\title{
1 Engineering SARS-CoV-2 cocktail antibodies into a bispecific format 2 improves neutralizing potency and breadth
}

4 Zhiqiang $\mathrm{Ku}^{1 *}$, Xuping $\mathrm{Xie}^{2 *}$, Jianqing $\mathrm{Lin}^{3 *}$, Peng Gao ${ }^{1 *}$, Abbas El Sahili ${ }^{3}$, Hang $\mathrm{Su}^{1}$, Yang $5 \mathrm{Liu}^{2}$, Xiaohua $\mathrm{Ye}^{1}, \mathrm{Xin} \mathrm{Li}^{1}$, Xuejun Fan ${ }^{1}$, Boon Chong Goh ${ }^{3,4}$, Wei Xiong ${ }^{1}$, Hannah Boyd ${ }^{1}$, 6 Antonio E. Muruato ${ }^{2}$, Hui Deng ${ }^{1}$, Hongjie Xia ${ }^{2}$, Zou Jing ${ }^{2}$, Birte K. Kalveram ${ }^{2}$, Vineet D.

7 Menachery $^{5}$, Ningyan Zhang ${ }^{1}$, Julien Lescar ${ }^{3 \dagger}$, Pei-Yong Shi ${ }^{2 \dagger}$, and Zhiqiang An ${ }^{1 \dagger}$

$9 \quad{ }^{1}$ Texas Therapeutics Institute, Brown Foundation Institute of Molecular Medicine, University 10 of Texas Health Science Center at Houston, Houston, TX 77030, USA

$11{ }^{2}$ Department of Biochemistry and Molecular Biology, Institute for Human Infection and 12 Immunity, Sealy Institute for Vaccine Sciences, Sealy Center for Structural Biology \& 13 Molecular Biophysics, Department of Pharmacology \& Toxicology, University of Texas 14 Medical Branch, Galveston, TX 77555, USA

$15{ }^{3}$ NTU Institute of Structural Biology and School of Biological Sciences, Nanyang 16 Technological University, 636921, Singapore

$17{ }^{4}$ Antimicrobial Resistance Interdisciplinary Research Group, Singapore-MIT Alliance for 18 Research and Technology Centre, 138602, Singapore

$19{ }^{5}$ Department of Microbiology \& Immunology, University of Texas Medical Branch, Galveston, 20 TX, 77555, USA

$22 *$ These authors contributed equally to this work.

$23 †$ Correspondence: Julien@ntu.edu.sg, peshi@UTMB.EDU and Zhiqiang.An@uth.tmc.edu 


\section{Abstract}

25 One major limitation of neutralizing antibody-based COVID-19 therapy is the requirement of costly cocktails to reduce antibody resistance. We engineered two bispecific antibodies (bs Abs) using distinct designs and compared them with parental antibodies and their cocktail. Single molecules of both bsAbs block the two epitopes targeted by parental antibodies on the receptorbinding domain (RBD). However, bsAb with the IgG-(scFv) 2 design (14-H-06) but not the CrossMAb design (14-crs-06) increases antigen-binding and virus-neutralizing activities and spectrum against multiple SARS-CoV-2 variants including the Omicron, than the cocktail. Xray crystallography and computational simulations reveal distinct neutralizing mechanisms for individual cocktail antibodies and suggest higher inter-spike crosslinking potentials by $14-\mathrm{H}-$ 06 than 14-crs-06. In mouse models of infections by SARS-CoV-2 and the Beta, Gamma, and Delta variants, 14-H-06 exhibits higher or equivalent therapeutic efficacy than the cocktail. Rationally engineered bsAbs represent a cost-effective alternative to antibody cocktails and a promising strategy to improve potency and breadth.

\section{Introduction}

40 The COVID-19 pandemic has ravaged the world with unprecedented health, social and 41 economic losses ${ }^{1}$. Vaccination is among the most effective countermeasures but not sufficient

42 to end the pandemic due to challenges such as limited global access, vaccine hesitancy, and 43 waning effectiveness against variants ${ }^{2-4}$. Effective treatments are necessary for the patients, 44 unvaccinated populations and immunocompromised people who cannot generate protective immunity after vaccination ${ }^{5}$.

Neutralizing antibodies have proved to be effective against COVID-19. The RBD of SARS-CoV-2 spike protein (S) directly contacts the cellular receptor angiotensin-converting enzyme 2 (ACE2). It is the target of the most potent neutralizing antibodies ${ }^{6}$. However, drug resistance rapidly arises with antibody monotherapies regardless of neutralizing potency and epitope conservation of the antibodies ${ }^{7}$. Emerging SARS-CoV-2 variants of concern (VOC), such as the Beta and Gamma, have evolved RBD mutations that escape from neutralization by many single antibodies and some combined antibodies with overlapping epitopes ${ }^{8}$. Rationally designed antibody cocktails, which cover non-overlapping epitopes, can reduce SARS-CoV-2 escape mutations and expand neutralizing coverage of emerging variants ${ }^{9,10}$. Three antibody 
55 cocktails have received approval for emergency use, and several candidates are in advanced

56 stages of clinical trials. Despite the encouraging progress, antibody cocktail approaches

57 increase manufacturing costs and require high dose infusion in patients ${ }^{11}$, making it challenging

58 to have a global impact on pandemic response ${ }^{12}$. Recently, a wide range of antibodies has

59 dramatically or completely lost neutralization against the Omicron variant ${ }^{13-16}$, making the FDA

60 to limit the use of two approved antibody cocktails.

Bi-specific antibodies (bsAbs) are an emerging drug modality designed to combine the binding specificities of two antibodies into one molecule. With different designs, bsAbs can be engineered into diverse formats with varied valencies. One attractive feature for bsAbs is their potential to display novel functionalities that do not exist in mixtures of parental antibodies ${ }^{17}$. For example, engineered HIV-1 neutralizing bsAbs in the CrossMAb format have enhanced virus-neutralizing potency and breadth compared with the mixtures of parental antibodies ${ }^{18,19}$. With the same CrossMAb design, a SARS-CoV-2 bsAb (CoV-X2) exhibits a neutralizing activity superior to one parental antibody and similar to the other parental antibody ${ }^{20}$, suggesting the need to test other bsAb designs for improvement of bs Ab functions. In vitro and in vivo comparisons of bsAbs with parental antibodies and the cocktail, which are lacking in previous studies, will provide more insights for developing efficacious bsAb-based COVID-19 therapeutics.

We have previously identified two SARS-CoV-2 neutralizing antibodies, called CoV2escape mutations as a cocktail ${ }^{21}$. In this study, we engineered the two antibodies into two bsAbs, one using the CrossMAb design and the other using the $\mathrm{IgG}-(\mathrm{scFv})_{2}$ design. Using biochemical,

78 CrossMAb design, enhances neutralizing potency and spectrum against multiple SARS-CoV2 variants in comparison with parental antibodies and the cocktail.

\section{Results}

\section{Engineering of bispecific antibodies}

83 We sought to construct bsAbs to combine the utility of CoV2-06 and CoV2-14 into one single 84 molecule. To explore whether and how the design of formats affect the functions of bsAbs, we 85 engineered two bsAbs with distinct features: one bsAb (14-H-06) is in the tetravalent format 
using the $\mathrm{IgG}-(\mathrm{scFv})_{2}$ design, and the other bsAb (14-crs-06) is in the bivalent format using the CrossMAb design (Fig. 1a). The two bsAbs were produced by transient expressions in Expi293F cells with high yields $(>500 \mu \mathrm{g} / \mathrm{ml})$. After a single-step Protein A chromatography purification, the bsAbs were showed $>95 \%$ purities and correctly assembled as analyzed by size-exclusion chromatography (SEC) (Fig. 1b). To test whether the bsAbs block the two epitopes targeted by CoV2-06 and CoV2-14, we performed an in-tandem Bio-Layer Interferometry (BLI) based binding assay (Fig. 1c). Both 14-H-06 and 14-crs-06 bound to RBD and blocked the subsequent binding of CoV2-06 and CoV2-14 (Fig. 1d). In contrast, pre-

\section{Enhanced antigen binding and virus neutralization for 14-H-06}

We characterized the antigen-binding properties of the two bsAbs and the two parental antibodies using BLI-based kinetic assays. To measure the affinity binding, we immobilized antibodies onto protein A biosensors and used soluble His-tagged RBD (RBD-His) as the analyte. Both bsAbs bound to RBD with affinity $\mathrm{K}_{\mathrm{D}}$ values in the low nanomolar range and comparable to the two parental antibodies (Extended Data Fig. 1a-b). The result is consistent with the intrinsic binding strength of the one-to-one interaction for the Fab, or scFv, to the RBD. To measure the avidity binding, we immobilized Ni-NTA biosensors with RBD-His at different concentrations and used antibodies as the analyte (Fig. 2a and Extended Data Fig. 1c-e). Avidity represents the combined strength of all binding sites on an antibody molecule and often manifests as decrease of dissociation from tethered antigens ${ }^{22}$. Accordingly, as the concentration of RBD for immobilization increased from $40 \mathrm{ng} / \mathrm{ml}$ to $1000 \mathrm{ng} / \mathrm{ml}$, the tetravalent antibody14-H-06 showed a greater increase of avidity binding ( $\mathrm{K}_{\mathrm{D}}$ values changed

111 from $1.35 \mathrm{nM}$ to $<0.001 \mathrm{nM}$, over 1350-fold) than the bivalent antibodies 14-crs-06 (8.6-fold 112 change), CoV2-06 (46-fold change) and CoV2-14 (2.0-fold change) (Fig. 2b). The increased 113 avidity binding was due to much slower dissociation of 14-H-06 from the RBD than other 114 antibodies, which were demonstrated by its larger fold changes of the $1 / \mathrm{K}_{\mathrm{dis}}$ values (Fig. 2c). 115 These results indicate that 14-H-06 enhances antigen binding activity with stronger avidity 116 effects than the 14-crs-06. 
We compared the neutralizing activities of the bsAbs and parental antibodies using authentic SARS-CoV-2 virus ${ }^{21}$. Antibody 14-crs-06 neutralized SARS-CoV-2 with a halfmaximal neutralizing titer $\left(\mathrm{NT}_{50}\right)$ of $0.132 \mu \mathrm{g} / \mathrm{ml}$, which was similar to CoV2-06 $\left(\mathrm{NT}_{50}=0.163\right.$ $\mu \mathrm{g} / \mathrm{ml})$ and 3.5-fold better than CoV2-14 $\left(\mathrm{NT}_{50}=0.462 \mu \mathrm{g} / \mathrm{ml}\right)$. This result is consistent with the trend that observed in CoV-X2, a previously reported SARS-CoV-2 bsAb with the same CrossMAb design ${ }^{20}$. In contrast, 14-H-06 neutralized SARS-CoV-2 with an NT50 of 0.032 $\mu \mathrm{g} / \mathrm{ml}$, which was 5.1-fold and 14.4-fold more potent than CoV2-06 and CoV2-14, respectively (Fig. 2d). To understand whether the two bsAbs alter the blocking activity against RBD binding to ACE2, we performed a BLI-based competition assay ${ }^{23}$. Antibody 14-H-06 blocked the RBD and ACE2 interaction with a half-maximal inhibition concentration $\left(\mathrm{IC}_{50}\right)$ of $9.0 \mathrm{nM}$, which is similar to the $\mathrm{IC}_{50}$ of $\mathrm{CoV} 2-14(10.6 \mathrm{nM})$ and slightly lower than the $\mathrm{IC}_{50 \mathrm{~s}}$ of 14 -crs-06 (21.2 nM) and CoV2-06 (39.8 nM) (Fig. 2e). These results indicate that the avidity binding, but not the steric hindrance with ACE2, contributes to the improvement of neutralizing activity for 14H-06 over 14-crs-06 and parental antibodies.

\section{Inter-spike crosslinking potential for 14-H-06}

133 The avidity binding of an antibody offers the opportunity to engage multiple spikes through crosslinking, which is an extra line of neutralizing mechanisms for certain RBD-targeting antibodies ${ }^{24}$. We sought to compare the potentials for inter-spike crosslinking by the bsAbs and 136 parental antibodies. In a BLI-based sandwich assay, RBD-His was immobilized onto Ni-NTA biosensors to capture antibodies, followed by incubation with Fc-tagged RBD (RBD-Fc). After RBD-His capturing, 14-H-06 showed much stronger binding to RBD-Fc than did 14-crs-06 and the two parental antibodies (Extended Data Fig. 2). The result indicates that $14-\mathrm{H}-06$ can engage more RBDs simultaneously than 14-crs-06 and parental antibodies through the four binding moieties, suggesting a higher potential for inter-spike crosslinking.

145 interactions established at the binding interface between Fab06 and RBD showed that VH 146 residues N32, W34 from CDR-H1, S55 from CDR-H2, and T104 from CDR-H3 interact with 147 RBD residues N450, K444, Y449 and R346 while the VL residues N33 from CDR-L1 and D52 
148 from CDR-L2 interact with RBD residues T345 and R346, respectively (Fig. 3a). The

149 interactions revealed by X-ray crystallography are fully consistent with epitope mapping results

150 reported in our previous study ${ }^{9}$. Next, we used the Fab06/RBD crystal structure (this work) to

151 perform a superposition with two cryo-EM structures of the spike trimer: one where the three

152 RBD adopt the down conformation and the other with one RBD up and two RBD down. As no

153 steric hindrance was observed, this superposition suggests that Fab06 could bind to RBDs

154 regardless of their down/up conformation in the spike trimer (Fig. 3b, left), which supports the

155 ability of inter-spike crosslinking for bispecific antibodies incorporating the CoV2-06

156 paratope ${ }^{24}$. Although we were able to obtain the structure of the free Fab14 (Extended Data

157 Table 1), so far, our attempts to use X-ray diffraction to determine a Fab14/RBD crystal

158 structure have not been successful and work with Cryo-EM to determine this structure is in 159 progress. Meanwhile, we used the X-ray structures of Fab14 and RBD for docking using 160 Haddock 2.4 guided by previous epitope mapping results ${ }^{9}$. Docking suggests that Fab14 can 161 only bind RBD in the up confirmation as, in the down conformation, Fab14 would clash with 162 an adjacent RBD domain (Fig. 3b, right). Interestingly, Fab06 has little while Fab14 has large 163 steric clash with ACE2 (Fig.3c). The binding epitopes and orientations indicate that the major 164 neutralizing mechanisms for individual cocktail antibodies are different: CoV2-06 through 165 crosslinking of spikes and CoV2-14 through ACE2 blocking. We used the molecular dynamics 166 (MD) method to model the structures of bsAbs and superposed them with RBDs in the spike. 167 The result shows that both bsAbs could simultaneously engage multiple RBDs in different spike 168 trimer (Fig. 3d). However, the maximum number of spikes can be crosslinked by the two bsAbs 169 were different when binding to RBDs adopting various combinations of up and down 170 conformations. As summarized in Fig. 3e, the tetravalent 14-H-06 can crosslink more spikes 171 than the bivalent 14-crs-06 and parental antibodies in all possible scenarios.

173 Broader coverage of variants by 14-H-06 than the cocktail

174 We previously identified neutralization-resistant mutation K444R for CoV2-06 and E484A for 175 CoV2-14 and generated SARS-CoV-2 viruses that contain K444R or E484A mutations ${ }^{9,23}$. The 176 K444R virus escaped from CoV2-06 but was neutralized by CoV2-14; the E484A virus escaped 177 from CoV2-14 but was neutralized by CoV2-06 (Extended Data Fig. 3a-b). While the two 178 bsAbs and the cocktail (CoV2-06+CoV2-14) neutralized both escaping viruses, their potencies 
179 were significantly different. The $\mathrm{NT}_{50 \text { s }}$ for 14-crs-06 against the K444R and E484A viruses

180 were $2.29 \mu \mathrm{g} / \mathrm{ml}$ and $0.83 \mu \mathrm{g} / \mathrm{ml}$, respectively, which were slightly less potent compared with 181 the $\mathrm{NT}_{50 \mathrm{~S}}$ of the cocktail against the K444R $(1.02 \mu \mathrm{g} / \mathrm{ml})$ and E484A $(0.59 \mu \mathrm{g} / \mathrm{ml})$ viruses. In 182 contrast, 14-H-06 neutralized the K444R and E484A viruses with $\mathrm{NT}_{50 \text { s }}$ of $0.23 \mu \mathrm{g} / \mathrm{ml}$ and $1830.096 \mu \mathrm{g} / \mathrm{ml}$, which were 4.4-fold and 6.1-fold more potent compared with the cocktail (Fig. 4a-b). Consistent with the neutralization results, the bsAbs bound to the K444R and E484A mutant RBD proteins, while CoV2-06 and CoV2-14 bound to E484A and K444R mutant RBDs, respectively (Extended Data Fig. 3c).

We focused on 14-H-06 and evaluated its neutralizing activities against seven SARSCoV-2 variants using the plaque reduction neutralization test (PRNT) or fluorescent focus reduction test (FFRNT). The complete spike gene from Alpha (B.1.1.7), Beta (B.1.351), Gamma (P.1), Kappa (B.1.617.1), Delta (B.1.617.2), Lambda (C.37), B.1.618 or the Omicron (B.1.1.529) variant was engineered into the backbone of an early clinical isolate USA-

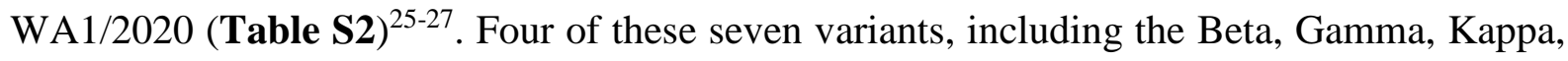
and the B.1.618 variants, carry the $\mathrm{E} 484 \mathrm{~K}$ or $\mathrm{E} 484 \mathrm{Q}$ mutation and were resistant to neutralization by CoV2-14 (Extended Data Fig. 3d). Notably, 14-H-06 potently neutralized all the tested variants with the $\mathrm{NT}_{50 \mathrm{~s}}$ between $0.009 \mu \mathrm{g} / \mathrm{ml}$ and $0.176 \mu \mathrm{g} / \mathrm{ml}$, which were in a close range compared with the $\mathrm{NT}_{50}(0.037 \mu \mathrm{g} / \mathrm{ml})$ against the US-WA1 strain (Fig. 4c). The Omicron (B.1.1.529) variant has 11 RBD mutations, including a G446S mutation within the CoV2-06 epitope and an E484A mutation within the CoV2-14 epitope. The two individual parental antibodies dramatically or almost completely lost neutralizing activity against the Omicron, while remarkably, 14-H-06 neutralized the Omicron with an $\mathrm{NT}_{50}$ of $1.11 \mu \mathrm{g} / \mathrm{ml}$, which is more than 10-fold more potent than the cocktail (Fig. 4d).

We further used a collection of 20 mutant RBD proteins to compare the epitope coverages by the two bsAbs, the cocktail, and individual parental antibodies through ELISA 205 variants or mutations in escaping viruses selected from two FDA approved antibodies: REGN20610987 and REGN-10933 ${ }^{23}$. Selected RBD mutations reduced the binding activities of 207 individual parental antibodies, such as K444R and K444Q mutations for CoV2-06 and E484A, 208 E484K, and F486V mutations for CoV2-14. Expectedly, the cocktail and the two bsAbs had 209 good coverages of these RBD variants (Fig. 4e). Across all the mutants, 14-H-06, but not 14- 
210 crs-06, exhibited significantly higher binding activities than the cocktail (Fig. 4f), indicating

211 that the IgG-(scFv) 2 design, but not the CrossMAb design, provides additional advantage for

212 binding to RBD mutants over the cocktail. Together, these data demonstrate that engineering

213 an antibody cocktail into a bsAb using the $\mathrm{IgG}-(\mathrm{scFv})_{2}$ design increases the neutralizing potency

214 against SARS-CoV-2 variants and broadens the epitope coverages of RBD mutants.

215

216 In vivo protection by 14-H-06

217 We focused on 14-H-06 to evaluate the in vivo efficacy against SARS-CoV-2 and its variants.

218 First, we performed dose range evaluations of 14-H-06 in the Balb/c mice infection model by 219 the CMA4 strain, a mouse-adapted SARS-CoV-2 containing the spike N501Y mutation which 220 represented the Alpha variant ${ }^{23}$ (Fig. 5a). Three dose levels (2.5, 0.83 and $0.27 \mathrm{mg} / \mathrm{kg}$ ) for 221 prophylactic treatment and two dose levels for therapeutic treatment ( 2.5 and $0.83 \mathrm{mg} / \mathrm{kg})$ were 222 tested. For prophylactic treatment, 14-H-06 reduced the viral loads in the lungs to undetectable 223 levels in 100\% (10/10) and 40\% (4/10) of mice in the 2.5 and $0.83 \mathrm{mg} / \mathrm{kg}$ groups, respectively. 224 Even with the $0.27 \mathrm{mg} / \mathrm{kg}$ dose, the geometric mean viral load (4.79-log) was 8.2-fold lower 225 than that from the isotype control group (5.70-log). For 14-H-06 therapy, the geometric mean 226 viral loads (excluding the mice with undetectable viruses) were reduced by 72,766- and 669227 fold in the 2.5 and $0.83 \mathrm{mg} / \mathrm{kg}$ groups, respectively (Fig. 5b). These data demonstrate that 14228 H-06 is highly effective for prophylactic and therapeutic treatment against SARS-CoV-2.

Next, we compared the therapeutic effects of 14-H-06, the cocktail, and individual parental antibodies against the CMA4 strain. The geometric mean viral load for the cocktail group was 1.79-log, significantly lower than was in the CoV2-14 group (4.21-log) and slightly

232 lower than was in the CoV2-06 group (2.51-log). In contrast, 14-H-06 showed substantially 233 better efficacy than the cocktail and individual parental antibodies, reducing viral loads to 234 undetectable levels for all mice (10/10) (Fig. 5c). We also compared the therapeutic effects of 235 14-H-06 and the cocktail against the Beta and Gamma variants in the Balb/c mouse model 236 following the same experimental design in Fig. 5a. Antibody 14-H-06 significantly reduced the 237 geometric mean lung viral loads by 136-fold for the Beta variant and 333-fold for the Gamma 238 variant compared with the isotype group. A slightly better efficacy against the Beta variant and 239 a more substantial better efficacy for 14-H-06 over the cocktail was observed against the Beta and Gamma variants, respectively (Fig. 5d-e). We further compared the therapeutic efficacy of 
14-H-06 and the cocktail against the Delta variant in the human ACE2 transgenic mouse (K18hACE2) model. We performed two sets of experiments to evaluate the therapeutic effects on viral replication (experiment set 1) and mice body weight change (experiment set 2) (Fig.5f). In experiment set 1 , we treated the mice with one dose of antibodies at $6 \mathrm{~h}$ after infection with the Delta variant, and the pfu assay measured viral loads in the lungs. Compared with the isotype group, 14-H-06 reduced the viral load by 278-fold, significantly more potent than the cocktail treatment, which reduced the viral load by 27.8-fold (Fig.5g). In experiment set 2, we treated mice at $6 \mathrm{~h}$ and $30 \mathrm{~h}$ after infection and monitored daily bodyweight. The viral loads were measured seven days post-infection (dpi) by qRT-PCR. The isotype-treated mice showed disease at day 7 post-infection, with an average of 14\% body weight loss (Fig. 5h) and the geometric mean viral RNA load of 7.2-log (Fig. 5i). Treatment with 14-H-06 and the cocktail significantly protected the mice from weight loss (Fig. 5h) and reduced the viral RNA loads in the lung (Fig. 5i). No significant difference in the lung viral RNA load was observed between 14-H-06 and the cocktail at day 7 (Fig. 5i).

Neutralizing antibody levels predict the protection from SARS-CoV-2 infection ${ }^{28}$. We performed a single dose $(10 \mathrm{mg} / \mathrm{kg})$ pharmacokinetics study in mice to compare the half-life of 14-H-06 with parental antibodies. The half-life for 14-H-06 was $29.2 \mathrm{~h}$ compared to $137.4 \mathrm{~h}$ and 74.72 h for CoV2-06 and CoV2-14, respectively (Extended Data Fig. 5a-b). Thus, the difference in the half-life may complicate the comparison of therapeutic efficacy, particularly in experiment set 2 for the Delta variant in the K18-hACE2 model. Taken together, these results demonstrate that 14-H-06 is superior or equivalent to the cocktail for therapeutic treatment of the original SARS-CoV-2 and subsequently emerged Beta, Gamma, and Delta variants in mice.

\section{Discussion}

Neutralizing antibody-based therapies are successful for treating viral infections, yet cocktails are often required to reduce resistance. We have shown that a SARS-CoV-2 bsAb offers advantages in neutralizing activities and spectrum against SARS-CoV-2 variants over the cocktail. Unlike bsAbs using the CrossMAb design, such as CoV-X2 $2^{20}$ and 14-crs-06, which do not or only slightly increase the neutralizing potency compared to parental antibodies or the cocktail, 14-H-06 significantly increases the neutralizing activity in vitro and therapeutic efficacy in vivo against SARS-CoV-2 and broadens the coverage of RBD variants. The IgG- 
$272(\mathrm{scFv})_{2}$ design outcompetes the CrossMAb design unlikely via stronger blockage of RBD 273 binding to ACE2, but rather via mechanisms including avidity binding and inter-spike 274 crosslinking. In support of our results, previous studies have shown that multivalent antibodies 275 have greater and broader neutralizing activity than bivalent $\mathrm{IgG}$ antibodies ${ }^{23,29}$. Similarly, a 276 SARS-CoV-2 tetravalent bsAb (CV1206_521_GS) uses the DVD-Ig design to combine the 277 RBD- and the NTD-specific antibodies, have demonstrated in vitro neutralizing activity that is 278 100-fold more potent than the cocktail. This DVD-Ig-based bsAb showed good neutralizing 279 coverages of several RBD mutations from some variants ${ }^{30}$; however, its neutralization potency 280 was compromised by the NTD mutations from the Beta and Gamma variants. Nevertheless, 281 rationally designed bsAbs with suitable formats and distinct epitope specificities represent a 282 promising alternative to antibody cocktails for developing COVID-19 therapeutic antibodies.

We directly compared the therapeutic efficacy of 14-H-06 and the cocktail against the spike N501Y mutation-containing CMA4 strain, the Beta, Gamma, and Delta variants in vivo. Across all these tested viruses, 14-H-06 has better efficacy than the cocktail regimen. These results support bs Abs as a promising alternative to cocktails for COVID-19 treatment. Although the $\mathrm{NT}_{50 \text { s }}$ of 14-H-06 against the US-WA1 strain, the Alpha, Beta and Gamma variants are in close range (less than 3-fold), 14-H-06 performed better against the CMA4 strain than the Beta and Gamma variants in vivo. Notably, antibody Fc-mediated effector functions are required for optimal therapeutic protections against SARS-CoV-2 in mice ${ }^{31}$. Antibody $14-\mathrm{H}-06$ is engineered using the $\mathrm{IgG}-(\mathrm{scFv})_{2}$ design, which is an effector function-competent format ${ }^{32}$.

292 However, it is possible that the effector functions of 14-H-06 have been compromised against 293 the Beta and Gamma variants as a result of the reduced binding for the two Fab14 units to the 294 E484K mutation-containing spikes. The two scFv06 units of 14-H-06 resist the E484K and 295 other mutations in the spike proteins of Beta and Gamma variants. Yet, it is unclear whether 296 the effector functions can be supported in this model of binding. Therefore, choosing an 297 antibody less affected by viral mutations as the IgG backbone for engineering the $\mathrm{IgG}-(\mathrm{scFv})_{2}$ 298 format of bsAbs may mitigate the risk of losing Fc-mediated effector functions. Systematic 299 investigation on whether and how bsAb designs affect the Fc-mediated effector functions will 300 provide further insights to guide the development of bsAb-based therapeutic antibodies against 301 SARS-CoV-2. 

more than ten-clinical stage bsAb candidates ${ }^{33}$. Antibody $14-\mathrm{H}-06$ expresses in high yield (>0.5 $\mathrm{g} / \mathrm{L})$ in transient expression and assembles homogenously, suggesting suitable early developmentability profiles. It is noted that 14-H-06 has a shorter half-life than the parental antibodies in mice, which may have limited its therapeutic advantage over the cocktail in vivo. The half-life of 14-H-06 may be extended by introducing the M252Y/S254T/T256E (YTE) mutations into the $\mathrm{Fc}$ region ${ }^{34}$, or by optimizing the antibody sequence toward favorable physical and chemical properties ${ }^{35}$; and the improved half-life could maximize the therapeutic potential of 14-H-06. The enhanced efficacy of 14-H-6 over the cocktail demonstrated in the in vitro and in vivo comparisons clearly support the potential to extend the application of the IgG$(\mathrm{scFv})_{2}$ design to other SARS-CoV-2 antibody cocktails.

In summary, we have engineered two formats of bsAbs and compared them with parental antibodies and the cocktail in a panel of in vitro and in vivo assays. Our results demonstrate the advantages of a bsAb design over the cocktail in neutralization potency and spectrum. This proof-of-concept study supports that the bsAb approach and the $\mathrm{IgG}-(\mathrm{scFv})_{2}$ design can be adapted to broader applications in the development of cost-effective and more efficacious antibody therapies on the basis of antibody cocktails for treating viral infections including SARS-CoV-2.

\section{References}

1 Dong, E., Du, H. \& Gardner, L. An interactive web-based dashboard to track COVID-19 in real time. The Lancet. Infectious diseases 20, 533-534, doi:10.1016/S1473-3099(20)30120-1 (2020).

2 Wouters, O. J. et al. Challenges in ensuring global access to COVID-19 vaccines: production, affordability, allocation, and deployment. Lancet 397, 1023-1034, doi:10.1016/S0140-6736(21)00306-8 (2021).

3 Koen B. Pouwels, E. P., Philippa C. Matthews, Nicole Stoesser, David W. Eyre, Karina-Doris Vihta, Thomas House, Jodie Hay, John I Bell, John N Newton, Jeremy Farrar, Derrick Crook, Duncan Cook, Emma Rourke, Ruth Studley, Tim Peto, lan Diamond, A. Sarah Walker, the COVID-19 Infection Survey Team. Impact of Delta on viral burden and vaccine effectiveness against new SARS-CoV-2 infections in the UK. medRxiv, doi:https://doi.org/10.1101/2021.08.18.21262237 (2021).

4 Machingaidze, S. \& Wiysonge, C. S. Understanding COVID-19 vaccine hesitancy. Nature medicine, doi:10.1038/s41591-021-01459-7 (2021).

5 Taylor, P. C. et al. Neutralizing monoclonal antibodies for treatment of COVID-19. Nature reviews. Immunology 21, 382-393, doi:10.1038/s41577-021-00542-x (2021).

6 Barnes, C. O. et al. SARS-CoV-2 neutralizing antibody structures inform therapeutic strategies. Nature 588, 682-687, doi:10.1038/s41586-020-2852-1 (2020).

7 Baum, A. \& Kyratsous, C. A. SARS-CoV-2 spike therapeutic antibodies in the age of variants. The Journal of experimental medicine 218, doi:10.1084/jem.20210198 (2021).

8 Montefiori, D. C. \& Acharya, P. SnapShot: SARS-CoV-2 antibodies. Cell host \& microbe 29, 1162-1162 e1161, doi:10.1016/j.chom.2021.06.005 (2021). 
Planas, D. et al. Considerable escape of SARS-CoV-2 Omicron to antibody neutralization. Nature, doi:10.1038/s41586-021-04389-z (2021). doi:10.1038/s41586-021-04388-0 (2021). Dejnirattisai, W. et al. Omicron-B.1.1.529 leads to widespread escape from neutralizing antibody responses. bioRxiv : the preprint server for biology, doi:10.1101/2021.12.03.471045 (2021). Cao, Y. et al. Omicron escapes the majority of existing SARS-CoV-2 neutralizing antibodies. Nature, doi:10.1038/s41586-021-04385-3 (2021).

17 Labrijn, A. F., Janmaat, M. L., Reichert, J. M. \& Parren, P. Bispecific antibodies: a mechanistic review of the pipeline. Nature reviews. Drug discovery 18, 585-608, doi:10.1038/s41573-019-0028-1 (2019). 1621-1631, doi:10.1016/j.cell.2016.05.024 (2016). Davis-Gardner, M. E., Alfant, B., Weber, J. A., Gardner, M. R. \& Farzan, M. A Bispecific Antibody That Simultaneously Recognizes the V2- and V3-Glycan Epitopes of the HIV-1 Envelope Glycoprotein Is Broader and More Potent than Its Parental Antibodies. mBio 11, doi:10.1128/mBio.03080-19 (2020). doi:10.1038/s41586-021-03461-y (2021).

Koenig, P. A. et al. Structure-guided multivalent nanobodies block SARS-CoV-2 infection and suppress mutational escape. Science 371, doi:10.1126/science.abe6230 (2021). pathogens 6, e1000908, doi:10.1371/journal.ppat.1000908 (2010).

$23 \mathrm{Ku}, \mathrm{Z}$. et al. Nasal delivery of an IgM offers broad protection from SARS-CoV-2 variants. Nature, doi:10.1038/s41586-021-03673-2 (2021).

Barnes, C. O. et al. Structures of Human Antibodies Bound to SARS-CoV-2 Spike Reveal Common Epitopes and Recurrent Features of Antibodies. Cell 182, 828-842 e816, doi:10.1016/j.cell.2020.06.025 (2020). doi:10.1038/s41586-021-03693-y (2021).

Liu, Y. et al. Neutralizing Activity of BNT162b2-Elicited Serum. The New England journal of medicine, doi:10.1056/NEJMc2102017 (2021).

Liu, Y. et al. BNT162b2-Elicited Neutralization against New SARS-CoV-2 Spike Variants. The New England journal of medicine 385, 472-474, doi:10.1056/NEJMc2106083 (2021).

28 Khoury, D. S. et al. Neutralizing antibody levels are highly predictive of immune protection from symptomatic SARS-CoV-2 infection. Nature medicine 27, 1205-1211, doi:10.1038/s41591-021-01377-8 (2021).

29 Miersch, S. et al. Tetravalent SARS-CoV-2 Neutralizing Antibodies Show Enhanced Potency and Resistance to Escape Mutations. Journal of molecular biology 433, 167177, doi:10.1016/j.jmb.2021.167177 (2021).

30 Cho, H. et al. Bispecific antibodies targeting distinct regions of the spike protein potently neutralize SARS-CoV-2 variants of concern. Science translational medicine, eabj5413, doi:10.1126/scitranslmed.abj5413 (2021).

31 Winkler, E. S. et al. Human neutralizing antibodies against SARS-CoV-2 require intact Fc effector functions for optimal therapeutic protection. Cell 184, 1804-1820 e1816, doi:10.1016/j.cell.2021.02.026 
(2021).

32 Brinkmann, U. \& Kontermann, R. E. The making of bispecific antibodies. mAbs 9, 182-212, doi:10.1080/19420862.2016.1268307 (2017).

33 Elshiaty, M., Schindler, H. \& Christopoulos, P. Principles and Current Clinical Landscape of Multispecific Antibodies against Cancer. International journal of molecular sciences 22, doi:10.3390/ijms22115632 (2021).

34 Dall'Acqua, W. F., Kiener, P. A. \& Wu, H. Properties of human IgG1s engineered for enhanced binding to the neonatal Fc receptor (FcRn). The Journal of biological chemistry 281, 23514-23524, doi:10.1074/jbc.M604292200 (2006).

35 Seeliger, D. et al. Boosting antibody developability through rational sequence optimization. $m A b s$, 505-515, doi:10.1080/19420862.2015.1017695 (2015).

36 Xie, X. et al. An Infectious CDNA Clone of SARS-CoV-2. Cell host \& microbe 27, 841-848 e843, doi:10.1016/j.chom.2020.04.004 (2020).

$37 \mathrm{Su}, \mathrm{H}$. et al. A potent bispecific neutralizing antibody targeting glycoprotein B and the $\mathrm{gH} / \mathrm{gL} / \mathrm{pUL128} / 130 / 131$ complex of human cytomegalovirus. Antimicrobial agents and chemotherapy, doi:10.1128/AAC.02422-20 (2020).

38 Schaefer, W. et al. Immunoglobulin domain crossover as a generic approach for the production of bispecific IgG antibodies. Proceedings of the National Academy of Sciences of the United States of America 108, 11187-11192, doi:10.1073/pnas.1019002108 (2011).

39 Kabsch, W. Integration, scaling, space-group assignment and post-refinement. Acta crystallographica. Section D, Biological crystallography 66, 133-144, doi:10.1107/S0907444909047374 (2010).

40 McCoy, A. J. et al. Phaser crystallographic software. Journal of applied crystallography 40, 658-674, doi:10.1107/S0021889807021206 (2007).

41 Smart, O. S. et al. Exploiting structure similarity in refinement: automated NCS and target-structure restraints in BUSTER. Acta crystallographica. Section D, Biological crystallography 68, 368-380, doi:10.1107/S0907444911056058 (2012).

42 Afonine, P. V. et al. Towards automated crystallographic structure refinement with phenix.refine. Acta crystallographica. Section D, Biological crystallography 68, 352-367, doi:10.1107/\$0907444912001308 (2012).

43 Emsley, P. \& Cowtan, K. Coot: model-building tools for molecular graphics. Acta crystallographica. Section D, Biological crystallography 60, 2126-2132, doi:10.1107/S0907444904019158 (2004).

44 van Zundert, G. C. P. et al. The HADDOCK2.2 Web Server: User-Friendly Integrative Modeling of Biomolecular Complexes. Journal of molecular biology 428, 720-725, doi:10.1016/j.jmb.2015.09.014 (2016).

45 Webb, B. \& Sali, A. Comparative Protein Structure Modeling Using MODELLER. Current protocols in protein science 86, 29 1-2 9 37, doi:10.1002/cpps.20 (2016).

46 Phillips, J. C. et al. Scalable molecular dynamics with NAMD. Journal of computational chemistry 26, 1781-1802, doi:10.1002/jcc.20289 (2005).

47 Humphrey, W., Dalke, A. \& Schulten, K. VMD: visual molecular dynamics. Journal of molecular graphics 14, 33-38, 27-38, doi:10.1016/0263-7855(96)00018-5 (1996).

48 Best, R. B. et al. Optimization of the additive CHARMM all-atom protein force field targeting improved sampling of the backbone phi, psi and side-chain chi(1) and chi(2) dihedral angles. Journal of chemical theory and computation 8, 3257-3273, doi:10.1021/ct300400x (2012).

49 Emsley, P., Lohkamp, B., Scott, W. G. \& Cowtan, K. Features and development of Coot. Acta crystallographica. Section D, Biological crystallography 66, 486-501, doi:10.1107/\$0907444910007493 (2010).

50 Muruato, A. et al. Mouse Adapted SARS-CoV-2 protects animals from lethal SARS-CoV challenge. bioRxiv: the preprint server for biology, doi:10.1101/2021.05.03.442357 (2021). 
444 Acknowledgments: We thank Dr. Georgina Salazar for her editorial support and Dr Chong 445 Wai Liew for advice. The MD simulations were performed on ASPIRE-1 of the National 446 Supercomputing Centre, Singapore (https://www.nscc.sg).

447 Funding: This work was supported in part by a Welch Foundation grant AU-0042-20030616 448 and Cancer Prevention and Research Institute of Texas (CPRIT) Grants RP150551 and 449 RP190561 (Z.A.); NIH grants HHSN272201600013C, AI134907, AI145617, and 450 UL1TR001439, and awards from the Sealy Smith Foundation, Kleberg Foundation, John S. 451 Dunn Foundation, Amon G. Carter Foundation, Gillson Longenbaugh Foundation, and 452 Summerfield Robert Foundation (P-Y.S.); and AcRF Tier 1 RG105/20 (J.L.).

453 Author contributions: Z.K., P.G., and Z.A. conceived the study. Z.K. identified the 454 neutralizing antibody cocktail. P.G., Z.K. and H.S. engineered and produced the bispecific 455 antibodies. X.X, Y.L., A.E.M., J.Z. and V.D.M. performed neutralization and mouse studies. 456 J.L., A.E.S. and B.C.G. performed structural studies. Z.K. and X.Y. generated the RBD mutant 457 proteins. X.L. and X.F. performed mouse PK study. W.X. prepared the Fab. H.D., and H.B. 458 provided support with cell culture and transfection. N.Z., J.L., P-Y.S. and Z.A. supervised the 459 study. Z.K. and P.G. wrote the manuscript with input from the team. X.X., J.L., X.Y., N.Z., 460 J.L., P-Y.S. and Z.A. reviewed and edited the manuscript.

461 Competing interests: The University of Texas System has filed a patent on the SARS-CoV-2 462 IgG antibodies and the reverse genetic system and reporter SARS-CoV-2. X.X., Z.K, N.Z., P463 Y.S, and Z.A. are listed as co-inventors of the patent application. Other authors declare no 464 competing interests.

465 Data availability: Data associated with figures are available from the corresponding authors 466 upon reasonable request. Structures and structure factors reported in this work have been 467 deposited with the PDB with accession codes 7WPH (Fab-06-RBD complex) and 7WPV (Fab468 14). Source data are provided with this paper. 


\section{Figures and legends}

a
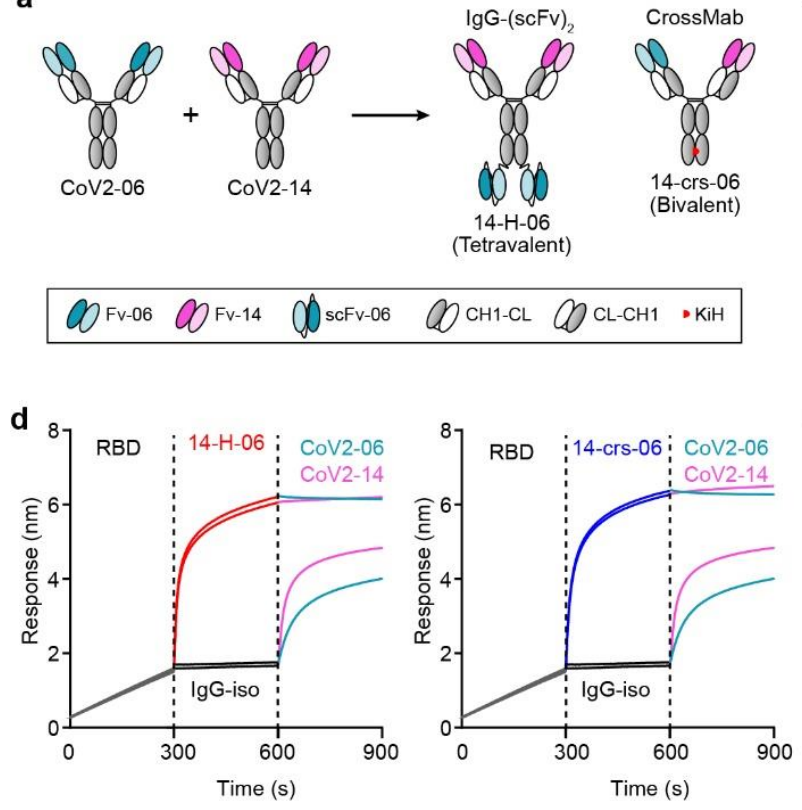

470

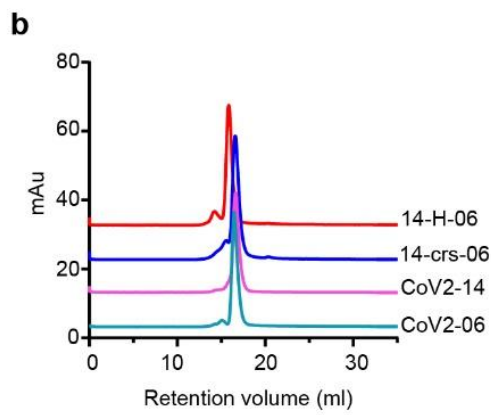

c

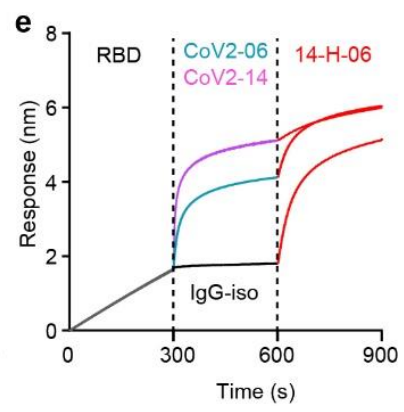

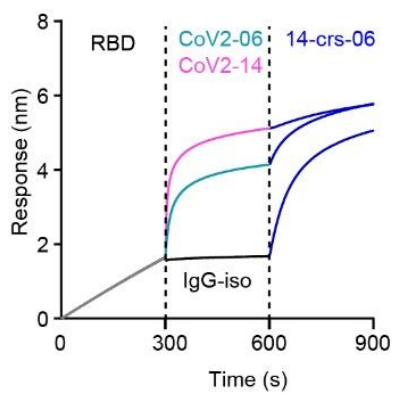

Fig. 1. Engineering of bispecific antibodies. a, Schematic diagram of engineering bispecific antibodies. A tetravalent bsAb (14-H-06) and a bivalent bsAb (14-crs-06) were engineered from two parental IgGs that bind to two distinct epitopes on the RBD. The modules for antibody engineering are illustrated in the box. Fv: variable fragment; scFv: single-chain Fv; CH1-CL: constant region 1 for heavy chain $(\mathrm{CH} 1)$ and constant region for light chain $(\mathrm{CL})$; CL-CH1: the crossover format of $\mathrm{CH} 1-\mathrm{CL}$; $\mathrm{KiH}$ : the Knob-into-Hole design with the S354C+T366W mutations (Knob) in the heavy chain $\mathrm{CH} 3$ region on one arm and the Y349C+T366S+L368A+Y407V mutations (Hole) in the $\mathrm{CH} 3$ region on the other arm. b, Purities of indicated antibodies were analyzed by SEC. c, The in-tandem BLI-based assay to test antibody blocking of RBD epitopes. The His-tagged RBD was captured onto the NiNTAbiosensors. The first antibodies, either bsAbs or parental antibodies, were used to bind the RBD. The second antibodies were tested for their abilities to bind pre-blocked RBD. d-e, The epitope pre-blocking effects using bispecific antibodies 14-H-06 (left) and 14-crs-06 (right) as $1^{\text {st }}$ antibodies and parental antibodies as $2^{\text {nd }}$ antibodies $(\mathbf{d})$, or using parental antibodies as $1^{\text {st }}$ antibodies and the bispecific antibodies 14-H-06 (left) and 14-crs-06 (right) as $2^{\text {nd }}$ antibodies (e). The segments of each sensorgram were color coded to show individual binding events. 
a
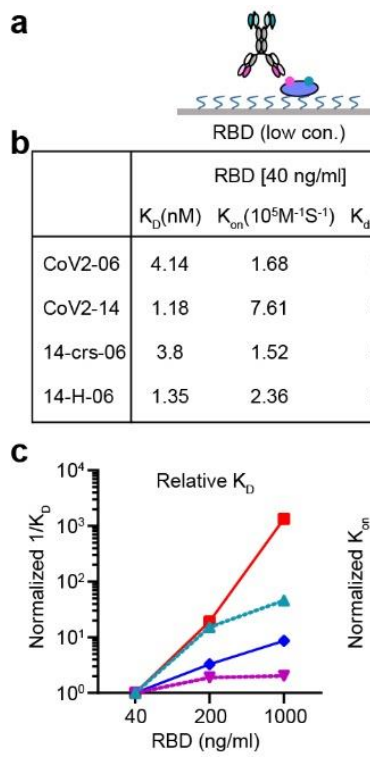

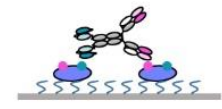

RBD (medium con.)

RBD [200 ng/ml]

$\begin{array}{lll}(\mathrm{nM}) & \mathrm{K}_{\text {on }}\left(10^{5} \mathrm{M}^{-1} \mathrm{~S}^{-1}\right) \mathrm{K}_{\text {dis }}(105 . \\ 0.27 & 2.15\end{array}$

69.5

57.7

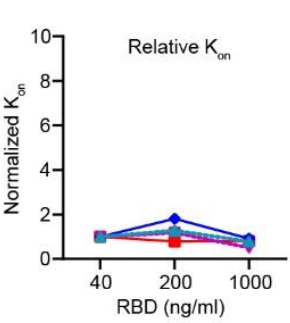

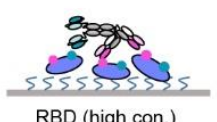

RBD [1000 ng/ml]

$K_{D}(n M) K_{o n}\left(10^{5} M^{-1} S^{-1}\right) K_{d i s}\left(10^{-5} S^{-1}\right)$

$\begin{array}{lll}0.09 & 1.33 & 1.26\end{array}$

$0.58 \quad 3.86$

$0.44 \quad 1.4$

$<0.001 \quad 1.91$

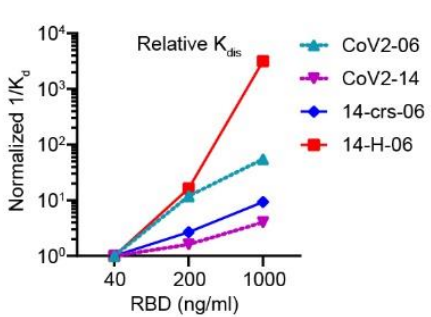

d
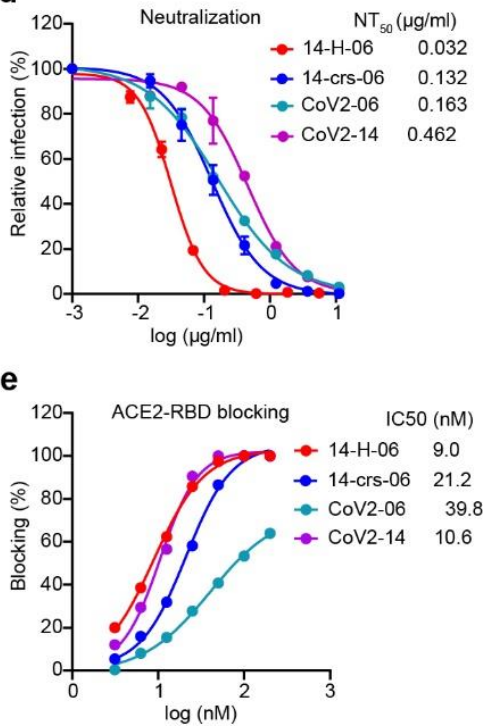

488

Fig. 2. Enhanced avidity binding and virus neutralization for 14-H-06. a, A diagram showing the binding models of 14-H-06 to tethered RBD antigen at low (left), medium (middle) and high (right) concentrations. The avidity effects manifest as multivalent interactions between an antibody and multiple RBDs. $\mathbf{b}$, Summary of the association $\left(\mathrm{K}_{\text {on }}\right)$, dissociation $\left(\mathrm{K}_{\mathrm{dis}}\right)$ and avidity $\left(\mathrm{K}_{\mathrm{D}}\right)$ of indicated antibodies at indicated concentrations RBD. $\mathbf{c}$, The plots of relative association, dissociation and avidity for each antibodies. The relative values for each antibodies were obtained by normalizing the values of $1 / \mathrm{K}_{\mathrm{D}}, \mathrm{K}_{\mathrm{on}}$ and $1 / \mathrm{K}_{\text {dis }}$ at $\mathrm{RBD}$ concentrations of 200 $\mathrm{ng} / \mathrm{ml}$ and $1000 \mathrm{ng} / \mathrm{ml}$ against the corresponding values at RBD concentration of $40 \mathrm{ng} / \mathrm{ml}$. d, Neutralization titrations of indicated antibodies against live SARS-CoV-2 on Vero E6 cell. Data points are from duplicate wells. e, Antibody blocking of RBD interaction with ACE2 determined by the BLI assay. 
a

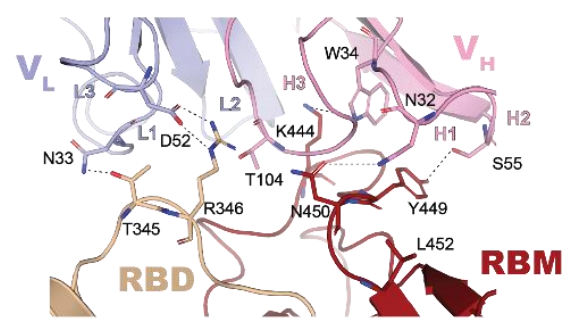

b

c ACE2

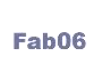

Fab06
RBD down

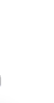

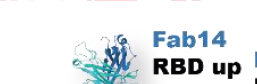

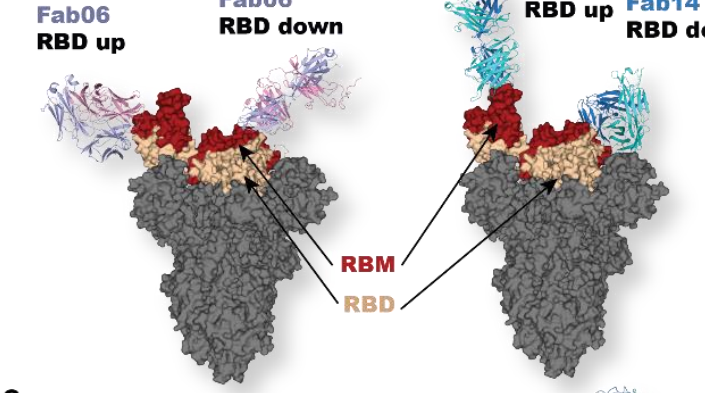

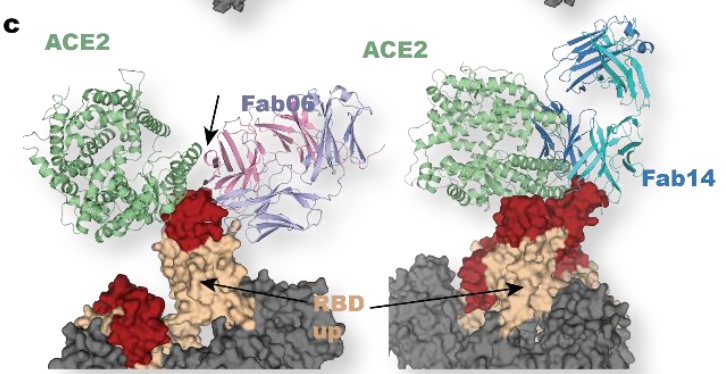

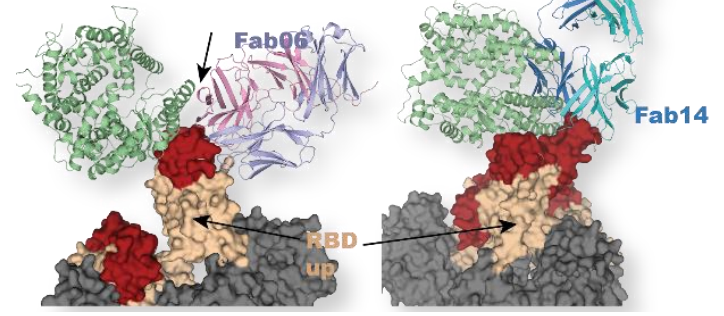

Spike trimer RBD up
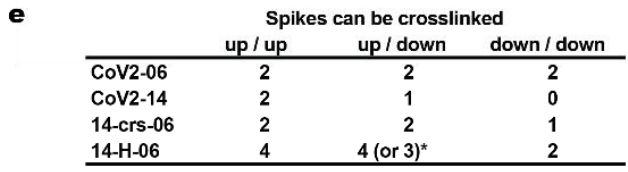

$14-\mathrm{H}-06$ 14-crs-06 binding to Spike trimers

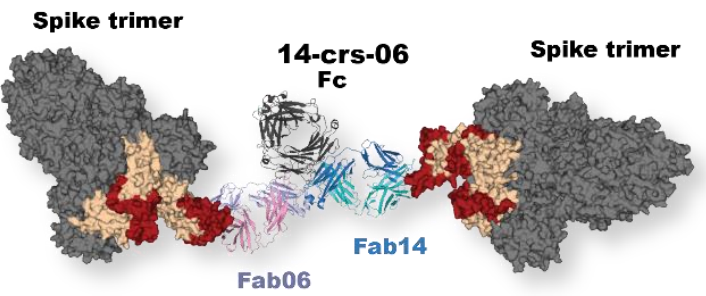

14-H-06 binding to Spike trimers

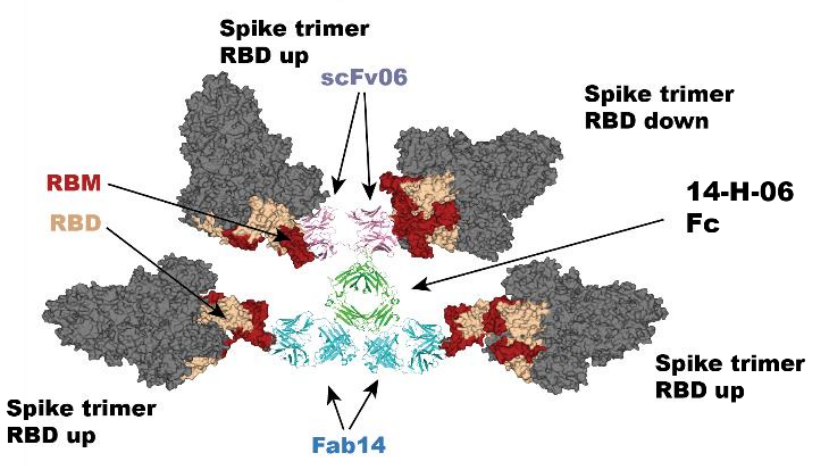

500

501

502

503

504

505

506

507

508

509

510

511

512

513

Fig. 3. Binding and inter-spike crosslinking potential for 14-H-06. a. Atomic details at the binding interface between Fab-06 and RBD as revealed by X-ray crystallography. The VH and VL of Fab-06 are colored in red and blue, respectively and RBD is colored in yellow. Dashed lines indicate polar interactions between Fab-06 and RBD. b. Binding models for Fab-06 (left) or Fab-14 to RBDs (either in up or down conformation) in the context of the complete spike trimer. c. Based on the epitope they recognize, both Fab-06 and Fab-14 introduce steric hindrance (indicated by arrows) with ACE2 binding to RBD in the up conformation. d, Crosslinks induced by the bispecific antibodies as derived by structural studies and MD simulations. upper panel: the bivalent14-crs-06 cross-links two spike trimers while the tetravalent 14-H-06 (lower panel) can crosslink up to four spikes. For 14-H-06, two scFvs from CoV2-06 are positioned at one end of the molecule, while two Fab units of CoV2-14 are located at the other end. e, Summary of cross-linking potentials by the antibodies reported in this work. *14-H-06 could crosslink three spikes if it binds to three RBDs in the down conformation and one RBD in the up conformation. Otherwise, it could crosslink four spikes. 
a

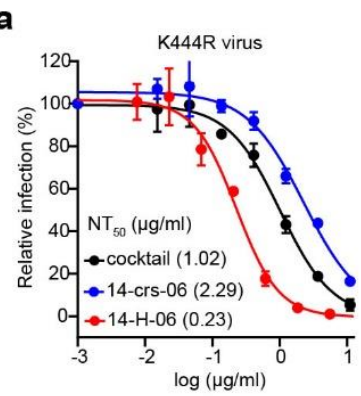

e
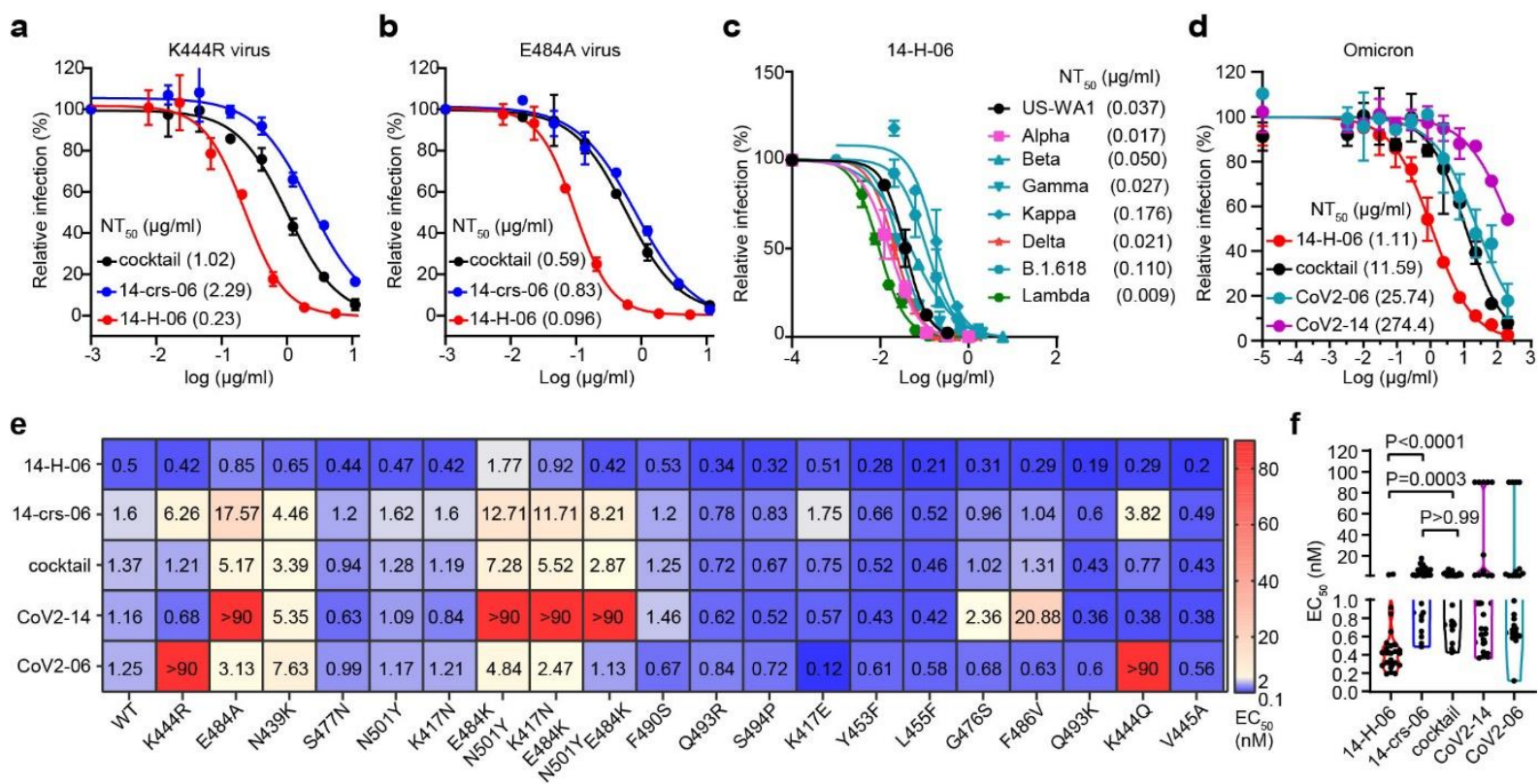

f

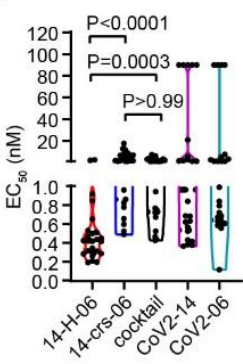

Fig. 4. Broad coverage of variants by 14-H-06. a-b, Neutralizations of the CoV2-06-resistant SARS-CoV-2 virus with K444R mutation (a) and CoV2-14-resistant SARS-CoV-2 virus with E484A mutation (b) by indicated bispecific antibodies and the antibody cocktail of CoV2-06 and CoV2-14. The assay is based on the mNeonGreen reporter virus and the $\mathrm{NT}_{50}$ values are labeled. c, Plaque reduction neutralization test (PRNT) of 14-H-06 against the SARS-CoV-2 US-WA1 strain and recombinant SARS-CoV-2 viruses with the spike replaced by those from indicated variants. The values of neutralizing titers $\left(\mathrm{NT}_{50}\right)$ are labeled. $\mathbf{d}$, Neutralizations of the Omicron variant by indicated bispecific antibodies, the antibody cocktail of CoV2-06 and CoV2-14 and two parental antibodies. The assay is based on the mNeonGreen reporter virus and the $\mathrm{NT}_{50}$ values are labeled. e, Summary of the ELISA binding $\mathrm{EC}_{50}$ values of indicated antibodies to the wild type RBD and a panel of 20 RBD mutants. $\mathbf{f}$, The violin plot of the $\mathrm{EC}_{50}$ values in d. The Kruskal-Wallis test was used for statistical analysis. 
a

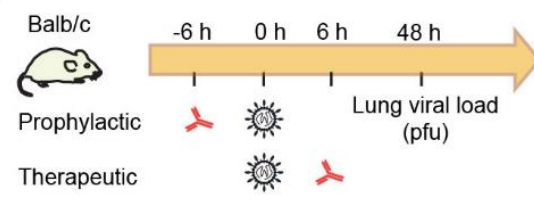

$\downarrow \operatorname{lgG}$ treatment (i.p) 济 (CMA4 strain, Beta or Gamma variants) b

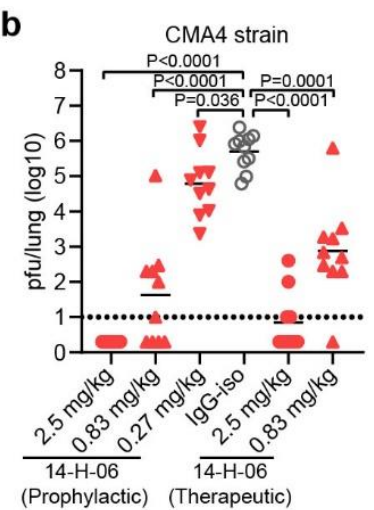

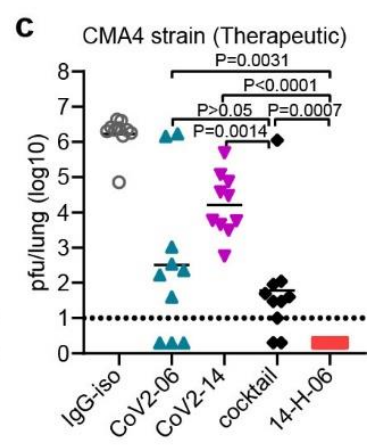

f
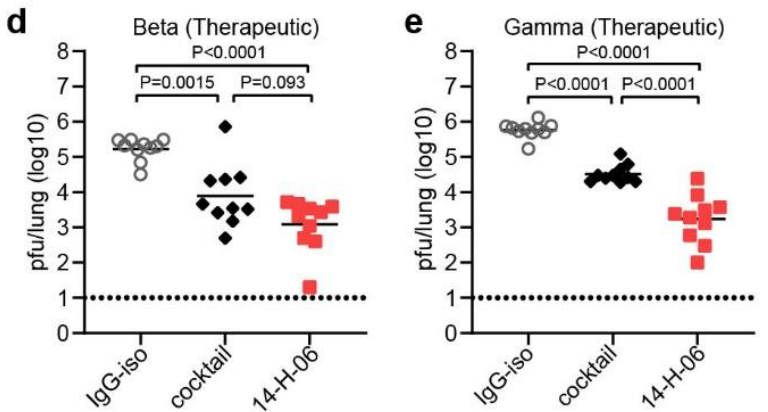

g

h

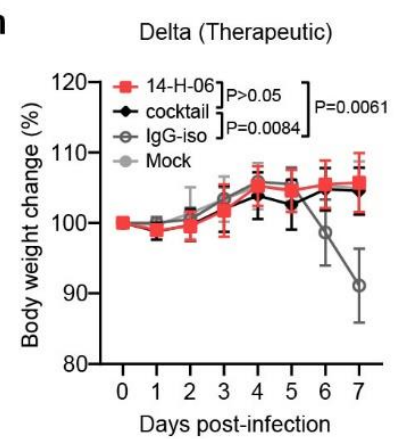

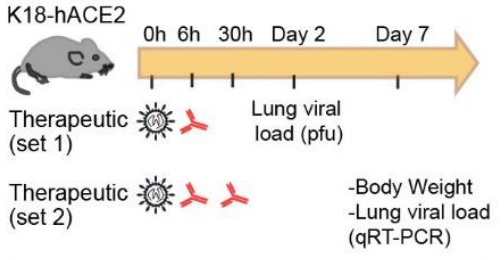

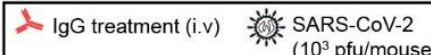
(Delta variant)

i

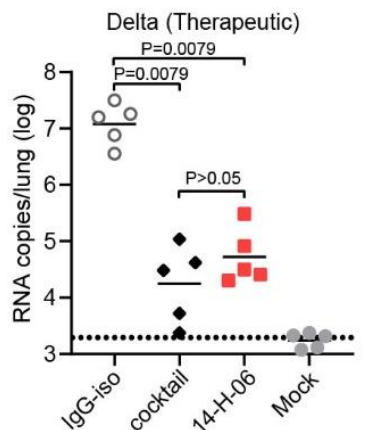

Fig. 5. In vivo comparisons of 14-H-06 and cocktail against SARS-CoV-2 and variants a, Experimental design for evaluations of the prophylactic and therapeutic effects of antibodies in the Balb/c mouse model of infections. Three SARS-CoV-2 viruses were tested, including a mouse-adapted CMA4 strain containing the spike N501Y mutation and representing the Alpha variant, and the Beta and Gamma variants. $n=10$ mice for each group. $\mathbf{b}$, The viral loads were determined by the pfu assay in the dose-range evaluations of the prophylactic and therapeutic effects of 14-H-06 against the CMA4 virus. c-e, The viral loads were determined by the pfu assay in the evaluations of the therapeutic effects of indicated antibodies at the dose of $5 \mathrm{mg} / \mathrm{kg}$ against the CMA4 virus (c), the Beta variant (d), and the Gamma variant (e). f, Experimental design for evaluating the therapeutic effect of 14-H-06 against the Delta variant in the transgenic K18-hACE2 mouse model. In experiment set 1, mice were treated with one dose of antibodies at $6 \mathrm{~h}$ after infection, and viral loads were measured at 2 days after infection by the pfu assay. In experiment set 2 , mice were treated with two doses of antibodies as indicated. The body weight was monitored daily, and the viral loads were measured at 7 days after infection by qRT-PCRR assay. $\mathrm{n}=10$ and 5 mice in each group for set 1 and set 2 , respectively. $\mathbf{g}$, the lung viral pfu loads for experimental set 1 in panel f. h-i, The body weight change (h), and the viral RNA load (i) for experiment set 2 in panel $\mathrm{f}$. In panels b-e, g, and i, the solid line indicates 
bioRxiv preprint doi: https://doi.org/10.1101/2022.02.01.478504; this version posted February 1, 2022. The copyright holder for this preprint (which was not certified by peer review) is the author/funder, who has granted bioRxiv a license to display the preprint in perpetuity. It is made available under aCC-BY-NC-ND 4.0 International license.

545 each group's geometric mean viral load, and the dotted line indicates the limit of detection 546 (LOD). For statistical analysis, the Mann-Whitney test was used in panels b-e, g, and i; the two547 way ANOVA with Tukey's multiple comparisons was used in panel h. 
548

549

550

551

552

553

554

\section{Material and methods}

\section{Cells, virus and proteins}

Expi293FTM cells (GIBCO, cat\#100044202) were maintained in Expi293 ${ }^{\text {TM }}$ Expression Medium without fetal bovine serum (FBS). Vero (ATCC ${ }^{\circledR}$ CCL-81) and Vero E6 cells (ATCC, CRL-1586) were maintained in Dulbecco's modified Eagle medium (DMEM) supplemented with $10 \%$ FBS. The wild-type and K444R and E484A mutations of mNeonGreen SARS-CoV2 viruses were generated as previously described ${ }^{36}$. The chimeric SARS-CoV-2 viruses with spike gene replaced with B1.1.7, P.1, and B.1.351 linage spike gene were described previously $^{23}$. The chimeric SARS-CoV-2 viruses with spike gene replaced with B.1.617.1, B.1.617.2, B.1.617.2-2, B.1.618 and the Omicron (B.1.1.529) linage spike gene were prepared from clinical strain USA-WA $1^{36}$. Summary of spike mutations of the variants were listed in Table S1.The biotinylated SARS-CoV-2 S protein was purchased from Acro Biosystem (Cat\# SPN-C82E9-25ug). The His-tagged RBD (RBD-His) protein of SARS-CoV-2 was purchased from Sino Biological (Cat: 40592-V08B). The Fc-tagged wild-type and mutant RBDs mentioned were generated as described previously ${ }^{23}$. The RBD for crystallography harbours a 8xHis tag and is fused to a Maltose Binding Protein via a TEV protease cleavage sequence and was produced from Expi293 ${ }^{\mathrm{TM}}$ cells. Protein purification was carried out in three steps: an IMAC purification using a HisTrap Ni-NTA column (Cytiva) followed by a TEV cleavage. A reverse IMAC purification was conducted to separate the MBP moiety from the soluble free RBD. The RBD was further purified by size-exclusion chromatography using a S200 16/60 column (Cytiva) pre-equilibrated in phosphate buffered saline at $\mathrm{pH}$ 7.2.

\section{Engineering and production of bsAbs}

Plasmids encoding heavy chain, light chain and scFv of CoV2-06 and CoV2-14 were constructed and described previously ${ }^{9}$. For engineering 14-H-06, a similar approach was used as described in a previous study ${ }^{37}$. Briefly, the scFv of CoV2-06 was fused to the C-terminus of CoV2-14 heavy chain with a $(\mathrm{G} 4 \mathrm{~S})_{3}$ linker to generate $14-\mathrm{H}-06$ heavy chain plasmid. The bsAb 14-H-06 was expressed by co-transfection of the modified heavy chain and the CoV2-14 light chain plasmids into Expi293F cells. For engineering of 14-crs-06, the CrossMab ${ }^{\mathrm{CH} 1-\mathrm{CL}}$ construct was used as described previously ${ }^{38}$. On one arm, the S354C and T366W mutations were introduced into the heavy chain $\mathrm{CH} 3$ region of $\mathrm{CoV}-06$ to generate the hole. This modified 
579 heavy chain was paired with the CoV2-06 light chain. On the other arm, the mutations Y349C,

580 T366S, L368A and Y407V mutations were introduced into the heavy chain CH3 region of CoV-

58114 with the crossover between the $\mathrm{CH} 1$ domain and the CL domain of the light chain of CoV2-

582 14. The 14-crs-06 antibody was expressed by co-transfection of four plasmids into Expi293F

583 cells. After 7 days of culture, antibodies were purified using the Protein A resin (Repligen, CA-

584 PRI-0100). All the antibody preparations were reconstituted in phosphate-buffered saline (PBS)

585 buffer for the studies. For the SEC assay, purified antibodies were analyzed on the ÄKTA pure

586 system with the Superpose 6 increase 10/300GL column in PBS buffer. About $100 \mu \mathrm{g}$ of each

587 antibody was used for each loading. The UNICORN 7.0 software was used to data analysis and

588 exporting.

\section{In-tandem BLI binding assays}

591 An in-tandem BLI-based binding assay was performed on the Pall ForteBio Octet RED96 592 system. The RBD-His $(1 \mu \mathrm{g} / \mathrm{ml})$ was loaded onto the Ni-NTA biosensors for 300 seconds. The 593 loaded biosensors were dipped into the first antibody solutions (400 nM) for 300 seconds for 594 the formation of the antibody-antigen complex. The sensors were then dipped into the second 595 antibody solutions (100 nM) for 300 seconds for competition binding. ForteBio's data analysis 596 software was used to export data, and the binding profile was processed by GraphPad prism 8 597 Software.

599 Antibody affinity and avidity assays

600 Kinetic analysis was performed using a Pall ForteBio Octet RED96 system. For the affinity 601 assays, antibodies were used as ligands to and loaded onto the Protein A biosensors, at $2 \mu \mathrm{g} / \mathrm{ml}$ 602 for 300s. Following 10s of baseline in kinetics buffer, the loaded biosensors were dipped into 603 serially diluted $(0.14-100 \mathrm{nM}) \mathrm{RBD}-$ His protein for 300 seconds for association. The sensors 604 were then dipped into a kinetic buffer for 600 seconds to record dissociation. Kinetic buffer 605 without antigen was set to correct the background. For the avidity assays, RBD-His was as 606 ligand and loaded onto the Ni-NTA biosensors at various concentrations (40, 200 and 1000 $607 \mathrm{ng} / \mathrm{ml}$ ) for 300s. Following 10s of baseline in kinetics buffer, the loaded biosensors were dipped 608 into serially diluted $(0.14-100 \mathrm{nM})$ antibodies 300 s for association, then dipped into kinetics 
609 buffer 400s for dissociation. ForteBio's data analysis software was used to fit the $\mathrm{K}_{\mathrm{D}}$ data using

610 the global fitting method.

\section{The BLI sandwich assay for testing multivalent binding to RBD}

613 The purified antibodies were tested for their abilities to simultaneously binding to multiple RBD 614 domains on the Octet RED96 system. The RBD-His $(5 \mu \mathrm{g} / \mathrm{ml})$ was captured on the Ni-NTA 615 biosensors for 300 seconds. After capture, the biosensors were dipped into antibody solutions 616 (200 nM) for 300 seconds, and finally to the RBD-Fc solution (200 nM) or PBS control for 300 617 seconds. The binding responses were recorded for all incubation steps. Last step association 618 (dissociation) was calculated by subtraction of PBS signal from the RBD-Fc binding.

\section{Crystallization}

621 The Fab-06 and RBD proteins were mixed in a 1:1.2 molar ratio and incubated on ice for 10 622 minutes, followed by size-exclusion chromatography using a S200 16/60 column (Cytiva) in 623 PBS. The complex peak was pooled and concentrated to $11 \mathrm{mg} / \mathrm{ml}$ for crystallization assays 624 which were set up with commercial screening kits (JCSG-plus ${ }^{\mathrm{TM}} \&$ Morpheus ${ }^{\circledR}$ from 625 Molecular Dimensions; Index ${ }^{\mathrm{TM}}$ \& PEG/Ion Screen ${ }^{\mathrm{TM}}$ from Hampton Research) using a 626 mosquito crystallization robot (TTP Labtech). A thin plate-shaped crystal was obtained in 627 JCSG-plus ${ }^{\mathrm{TM}}$ condition A5 (0.2 M magnesium formate dihydrate, 20\% w/v PEG 3350) with a 628 protein to buffer ratio of $2: 1$ after 13 days. The crystal was subsequently fished with a nylon 629 loop and flash-frozen in liquid nitrogen and shipped to synchrotron for remote data collection 630 (MXII, ANSTO's Australian Synchrotron). X-ray diffraction images were integrated and scaled 631 using $\mathrm{XDS}^{39}$. Molecular replacement was done via Phaser ${ }^{40}$ using $\mathrm{Fv}, \mathrm{CH}_{1} / \mathrm{C}_{\mathrm{L}}$, and $\mathrm{RBD}$ from 632 PDB accession code 7C01 as three search components. Structure refinement was performed 633 using both Buster ${ }^{41}$ and Phenix Refine ${ }^{42}$ interspersed with manual model correction using $634 \operatorname{Coot}^{43}$. Complex between Fab14 and RBD proteins were also prepared and set up for 635 crystallization in the same manner. Crystals were obtained in $0.1 \mathrm{M}$ Lithium Chloride, 30\% 636 (w/v) PEG 4000 with a protein to buffer ratio of $2: 1$ after 7 days. However only Fab14 was 637 present in the crystal. Data collection and refinement statistics for the Fab06-RBD complex and 638 free Fab14 crystal structure are presented in Table S1. Both structures were deposited on Protein 639 Data Bank with accession number 7WPH (Fab-06-RBD complex) and 7WPV (Fab-14). 


\section{Antibody blocking of RBD and ACE2 interaction}

642 The Fc-tagged RBD proteins $(4 \mu \mathrm{g} / \mathrm{ml})$ were captured on the protein A biosensor for $300 \mathrm{~s}$. Then, 643 the sensors were blocked by a control Fc protein $(150 \mu \mathrm{g} / \mathrm{ml})$ for 200 s to occupy the free protein 644 A on the sensor. The serially diluted antibodies $(0.041 \sim 30 \mathrm{nM})$ were then incubated with the 645 sensors for 200s to allow antibody and RBD binding. After 10s of baseline in kinetics buffer, 646 the sensors were dipped in to the ACE2 solution $(10 \mu \mathrm{g} / \mathrm{ml})$ for $200 \mathrm{~s}$ to record the response 647 signal. For analysis of the $\mathrm{IC}_{50}$ of the blocking, the ACE2 response values were normalized to 648 the starting points. The blocking percentages at each concentrations were calculated as: 649 (normalized ACE2 response of isotype antibody- normalized ACE2 response of tested 650 antibody)/ normalized ACE2 response of isotype antibody *100. The dose-blocking curves 651 were plotted and the blocking $\mathrm{IC}_{50}$ values were calculated by nonlinear fit in the GraphPad 652 prism 8 Software.

\section{Neutralization assays}

655 All SARS-CoV-2 manipulations were conducted at the Biosafety Level-3 facility with the 656 approval from the Institutional Biosafety Committee at the University of Texas Medical Branch. 657 The neutralizing activities of antibodies against SARS-CoV-2 and two escape mutant strains 658 (K444R and E484A) were measured as previously described using mNeonGreen (mNG) 659 reporter viruses ${ }^{23}$. Briefly, $1.2 \times 10^{4}$ Vero cells were plated into each well of a black clear flat660 bottom 96-well plate (Greiner Bio-One; Cat\# 655090). The cells were incubated overnight at $37{ }^{\circ} \mathrm{C}$ with $5 \% \mathrm{CO}_{2}$. On the following day, serially diluted antibodies were mixed with an equal 662 volume of virus. After $1 \mathrm{~h}$ incubation at $37^{\circ} \mathrm{C}$, the antibody-virus complexes were inoculated 663 into Vero cell plates with the final MOI of 2 . At $20 \mathrm{~h}$ post-infection, nuclei were stained by the addition of Hoechst 33342 to a final concentration of $10 \mu \mathrm{M}$. Fluorescent images were acquired

665 using a Cytation 7 multimode reader (BioTek). Total cells (in blue) and mNG-positive cells (in 666 green) were counted, and the infection rate was calculated. The relative infection rates were 667 calculated by normalizing the infection rate of each well to that of control wells (no antibody 668 treatment).

669 The neutralizing activities of antibodies against SARS-CoV-2 variants were measured using the plaque reduction neutralization test ${ }^{23}$. Briefly, antibodies were 3 -fold serially diluted 
671 and incubated with 100 plaque forming unit (PFU) of USA-WA1/2020 or mutant SARS-CoV-

672 2. After $1 \mathrm{~h}$ incubation at $37^{\circ} \mathrm{C}$, the antibody-virus mixtures were inoculated onto a monolayer

673 of Vero E6 cells pre-seeded on 6-well plates on the previous day. After $1 \mathrm{~h}$ of infection at $37^{\circ} \mathrm{C}$,

$6742 \mathrm{ml}$ of $2 \%$ SeaPlaque ${ }^{\mathrm{TM}}$ Agarose (Lonza) in DMEM containing 2\% FBS and $1 \%$

675 penicillin/streptomycin (P/S) was added to the cells. After 2 days of incubation, $2 \mathrm{ml}$ of $2 \%$

676 SeaPlaque ${ }^{\mathrm{TM}}$ Agarose in DMEM containing 2\% FBS, 1\% P/S and 0.01\% Neutral Red (Sigma)

677 were added on top of the first layer. After another $16 \mathrm{~h}$ of incubation at $37^{\circ} \mathrm{C}$, plaque numbers

678 were counted. The dilution concentration that suppressed $50 \%$ of viral plaques was defined as

679 PRNT $_{50}$.

680

\section{Molecular docking and MD simulations}

682

An intial model for the CoV2-14 scFv-RBD complex was obtained using the HADDOCK 2.4 webserver $^{44}$ by providing the experimental Fab-CoV2-14 structure (this work) and the RBD (PDB access code: 7CJF) X-ray structures as input. An atomic model for the tetravalent bsAb 14-H-06 was built by placing one CoV2-06 scFv molecule at each of the C-terminal ends of the CoV2-14 IgG molecule. A $\left(\mathrm{G}_{4} \mathrm{~S}\right)_{3}$ linker was then added using MODELLER ${ }^{45}$ to connect each of these CoV2-06 scFv to the $\mathrm{CH} 3$ domains of the CoV2-14 IgG. The initial model for the complex between 14-H-06 with four RBD molecules (one RBD bound for each of the four paratopes of IgG-scFv bsAb 14-H-06) was subjected to MD simulations using NAMD 2.12 The system was simulated in a water box where the minimal distance between the solute and the box boundary was $20 \AA$ along all three axes. The charges of the solvated system were neutralized with counter-ions, and the ionic strength of the solvent was set to $150 \mathrm{mM} \mathrm{NaCl}$ using $\mathrm{VMD}^{47}$. The final system contains over 1.2 million atoms, including proteins, water molecules, and ions. It was subjected to conjugate gradient minimization for 10,000 steps, subsequently heated to $310 \mathrm{~K}$ in steps of $5 \mathrm{ps}$. The system was equilibrated for $5 \mathrm{~ns}$ with the backbone atoms constrained by a harmonic potential of the form $\mathrm{U}(\mathrm{x})=\mathrm{k}\left(\mathrm{x}-\mathrm{x}_{\mathrm{ref}}\right)^{2}$, where $\mathrm{k}$ is 1

$697 \mathrm{kcal} \mathrm{mol}^{-1} \AA^{-2}$ and $\mathrm{X}_{\text {ref }}$ is the initial atom coordinates. The equilibrated system was simulated for 69850 ns under the NPT ensemble assuming the CHARMM36 force field for the protein ${ }^{48}$ and 699 assuming the TIP3P model for water molecules. Structure analysis and image production were 700 made using PyMOL (https://pymol.org, Schrödinger Inc.) and COOT ${ }^{49}$. 


\section{ELISA binding titrations of antibodies to RBD mutants}

703 The RBD proteins were coated on Corning high binding assay plates with a concentration of 2 $704 \mu \mathrm{g} / \mathrm{ml}$ at $4^{\circ} \mathrm{C}$ overnight and blocked with $5 \%$ skim milk at $37^{\circ} \mathrm{C}$ for 2 hours. Serially diluted 705 antibodies were added at a volume of $100 \mu \mathrm{l}$ per well for incubation at $37^{\circ} \mathrm{C}$ for $2 \mathrm{~h}$. The anti706 human IgG Fab2 HRP-conjugated antibody was diluted 1:5000 and added at a volume of 100 $707 \mu \mathrm{l}$ per well for incubation at $37^{\circ} \mathrm{C}$ for $1 \mathrm{~h}$. The plates were washed 5 times with PBST $(0.05 \%$ 708 Tween-20) between incubation steps. TMB substrate was added $100 \mu 1$ per well for color 709 development for 3 mins and $2 \mathrm{M} \mathrm{H}_{2} \mathrm{SO}_{4}$ was added $50 \mu 1$ per well to stop the reaction. The OD 450 710 was read by a SpectraMax microplate reader. The data points were plotted using GraphPad 711 Prism8, and the $\mathrm{EC}_{50}$ values were calculated using a three-parameter nonlinear model.

\section{Mouse infection models}

714 The animal study was carried out in accordance with the recommendations for care and use of 715 animals by the Office of Laboratory Animal Welfare, National Institutes of Health. The 716 Institutional Animal Care and Use Committee (IACUC) of University of Texas Medical Branch 717 (UTMB) approved the animal studies under protocol 1802011. A previously described mouse 718 infection model was used to evaluate antibody protection. Female BALB/c mice aged 10-12 719 weeks $(\mathrm{n}=10)$ were infected intranasally (IN) with $10^{4}$ PFU of mouse-adapted SARS-CoV-2 720 CMA4 strain $^{50}$ or the Beta and Gamma variants ${ }^{23}$ in $50 \mu 1$ of PBS. Animals were injected 721 intraperitoneally (i.p.) with antibodies 6 hours before or 6 hours after viral infection. Two days 722 after infection, lung samples of infected mice were harvested and homogenized in $1 \mathrm{ml}$ PBS 723 using the MagNA Lyser (Roche Diagnostics). The homogenates were clarified by 724 centrifugation at $15,000 \mathrm{rpm}$ for $5 \mathrm{~min}$. The supernatants were collected for measuring 725 infectious viruses by plaque assay on Vero E6 cells.

726 For mouse study with the Delta variant, the 8-10-week-old female K18-hACE2 mice 727 were ordered from The Jackson Laboratory. In experiment set 1, the mice were infected 728 intranasally with $10^{3}$ PFU of SARS-CoV-2 Delta spike variant (ref: NT162b2-elicited 729 neutralization of B.1.617 and other SARS-CoV-2 variants. Nature 596, 273-275 (2021).) in 50 $730 \mu$ of PBS. Animals were injected intraperitoneally (i.p.) with antibodies 6 hours and 30 hours 731 after viral infection. The body weight of each mouse was monitored daily. Seven days after 732 infection, lung samples of infected mice were harvested and homogenized in $1 \mathrm{ml}$ PBS for qRT- 
733 PCR analysis as indicated in a previous study ${ }^{23}$. In experiment set 2, the mice were infected 734 intranasally with $10^{4}$ PFU of SARS-CoV-2 Delta variant in $50 \mu$ of PBS. Animals were 735 injected intraperitoneally (i.p.) with antibodies 6 hours after viral infection. The body weight 736 of each mouse was monitored daily. Two days after infection, mouse lung samples were 737 harvested and homogenized in $1 \mathrm{ml}$ PBS for plaque assay as described previously ${ }^{23}$.

\section{The pharmacokinetics of antibodies in mice}

740 Animal experimental protocols were approved by the Animal Welfare Committee at the 741 University of Texas Medical School at Houston. Seven-week-old female BALB/c (Jackson lab, 742 USA) were randomly divided into three groups (5 mice/group) and were injected by i.p with $74310 \mathrm{mg} / \mathrm{kg}$ of antibody. After injection, mouse blood were collected at 4, 8, 24, $72 \mathrm{~h}$, and day 5, 744 day 7, and day 10. Mouse tail vein was used for blood collection, and up to $0.01 \mathrm{ml}$ of serum 745 was needed for quantification by ELISA. The mouse blood was collected using a sterile scalpel 746 blade, nick the lateral tail vein. Mouse blood (2-3 drops) were collected into Eppendorf tubes.

747 For mouse serum collection, the blood samples were stored at room temperature for 1 hour, and 748 then centrifuged the samples for $30 \mathrm{~min}$ at $15,000 \mathrm{rpm}$ at $4^{\circ} \mathrm{C}$. The mouse serum was carefully 749 transferred to the new 0.5-ml Eppendorf tubes, and stored them at $-20^{\circ} \mathrm{C}$ until assay. The 750 indirect ELISA was used to quantify serum antibody levels. Briefly, the 96-well plates were 751 coated with the wild type RBD antigen for quantitation of CoV2-06 and CoV2-14 752 concentrations, and the E481A RBD antigen for quantitation of 14-H-06. Antigens were coated 753 at the concentration of $2 \mu \mathrm{g} / \mathrm{ml}$ in PBS ( $\mathrm{pH}$ 7.2) and incubated at $4{ }^{\circ} \mathrm{C}$ overnight. Plates were 754 blocked with PBS supplemented with 3\% BSA at room temperature for $1 \mathrm{~h}$. The mouse sera 755 were diluted 400x for incubation for with plates for $2 \mathrm{~h}$ at room temperature. The HRP756 conjugated goat anti-human $\operatorname{IgG}-\mathrm{F}\left(\mathrm{ab}^{\prime}\right) 2$ was used as the secondary antibody and incubated at 757 room temperature for $1 \mathrm{~h}$. The plate washing, color development steps were the same as 758 described above in ELISA titrations. For analysis of the half-life, the Phoenix 64 WinNonlin 759 (8.3.3.33) software (Certara) was used according to instructions. 


\section{Extended Data Figures}
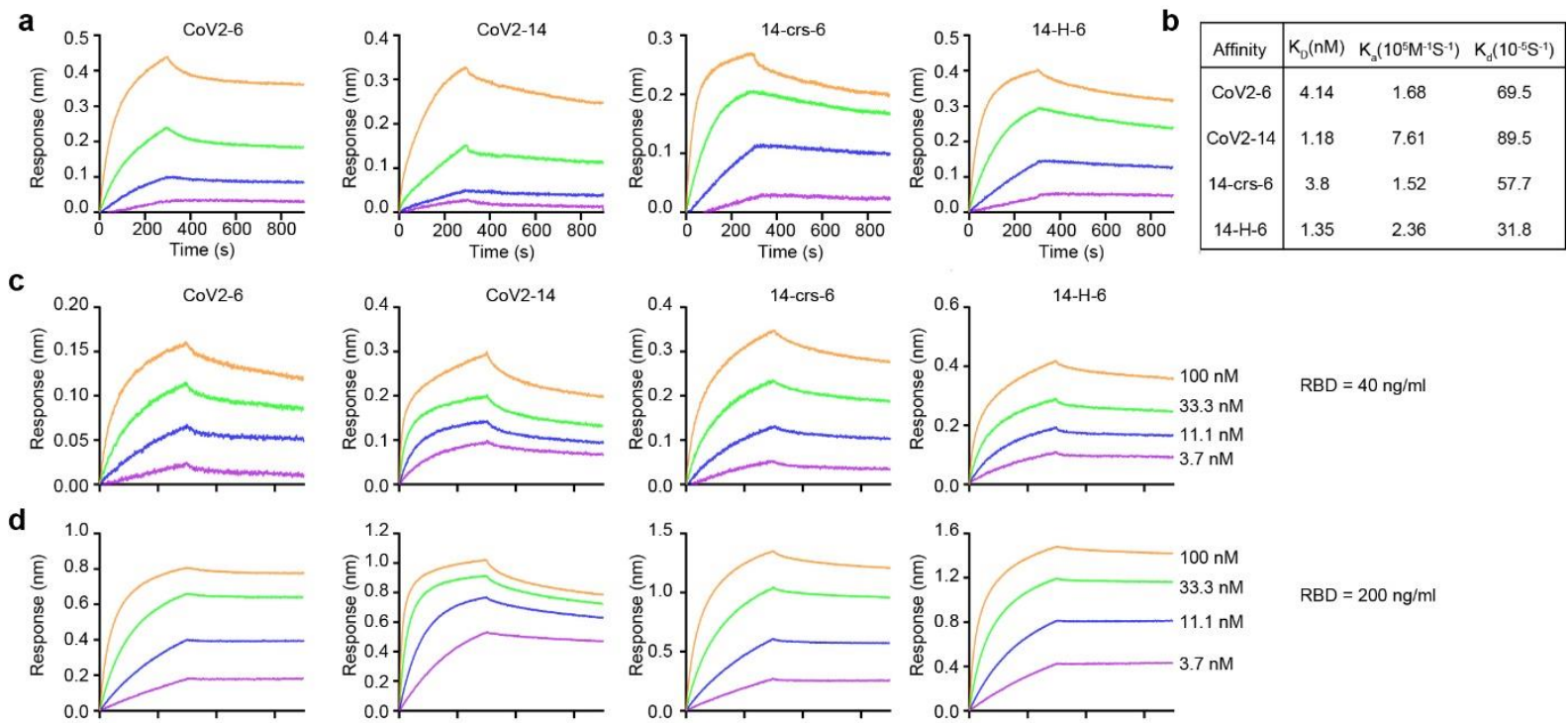

$\mathrm{RBD}=200 \mathrm{ng} / \mathrm{ml}$
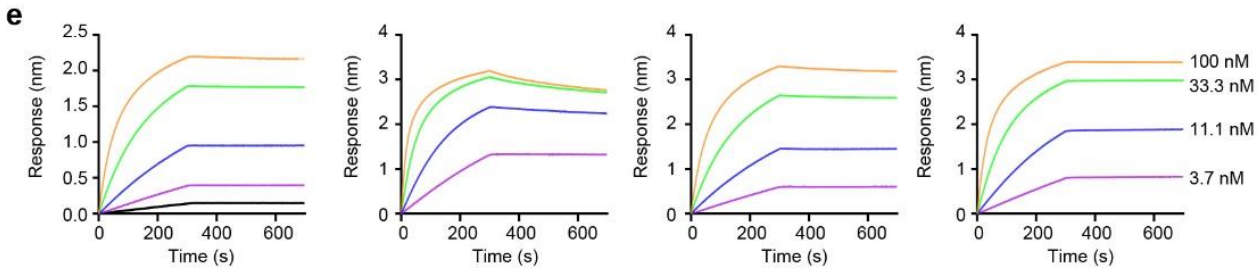

$\mathrm{RBD}=1000 \mathrm{ng} / \mathrm{ml}$

Extended Data Fig. 1. Kinetic bindings of the BLI-based affinity and avidity assays. a, The sensorgrams of affinity binding for indicated antibodies. Antibodies were immobilized onto the protein A biosensors and the RBD-His was in solutions. b, Summary of the affinity binding $\left(\mathrm{K}_{\mathrm{D}}\right)$, the association $\left(\mathrm{K}_{\mathrm{on}}\right)$ and the dissociation $\left(\mathrm{K}_{\mathrm{dis}}\right)$ parameters. c-e, The sensorgrams in the avidity binding for indicated antibodies. The RBD-His was immobilized onto the Ni-NTA biosensors at concentrations of $40 \mathrm{ng} / \mathrm{ml} \mathrm{(c),} 200 \mathrm{ng} / \mathrm{ml}$ (d) and $1000 \mathrm{ng} / \mathrm{ml}$ (e) and indicated antibodies were in solutions. 
a
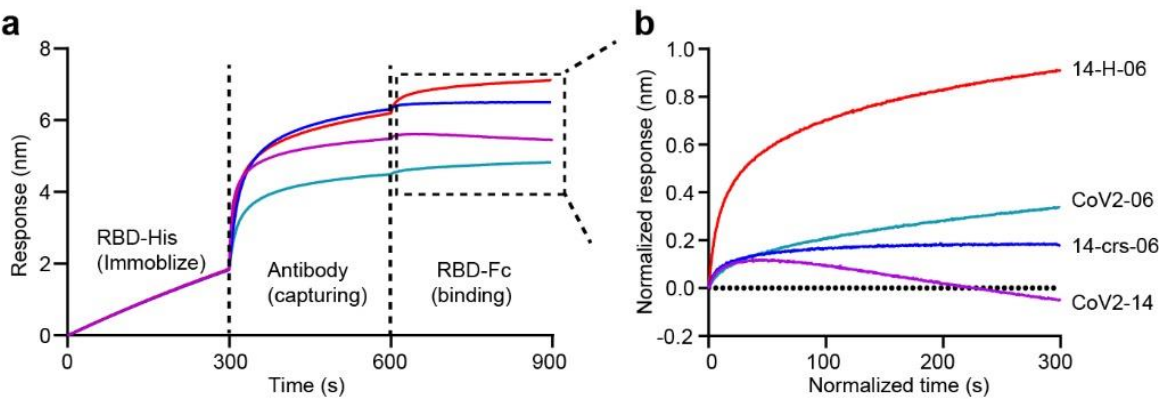

770 Extended Data Fig. 2. Multivalent binding to RBD by 14-H-06. Simultaneous binding of

771 antibodies to multiple RBDs was determined by a BLI sandwich assay. a, The sensorgrams

772 showing the immobilization of RBD-His for 300s, the capturing of indicated antibodies for

773 300s and the following binding by RBD-Fc for 300s. The binding to RBD-Fc shown in the

774 dashed box was normalized and shown in the panel $\mathbf{b}$. 

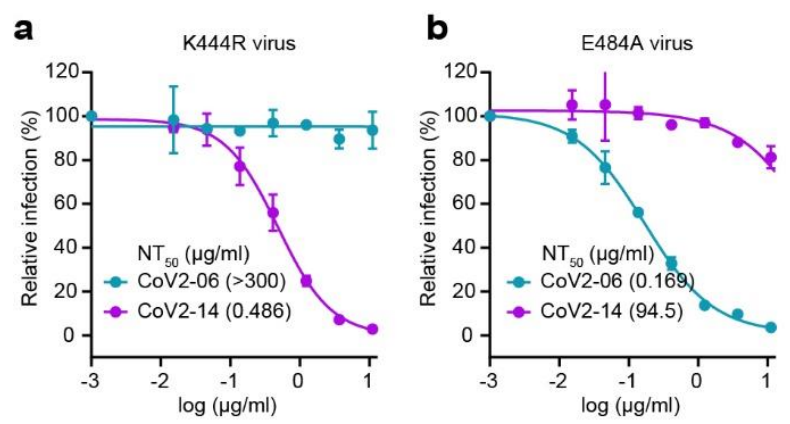

C

\section{d}
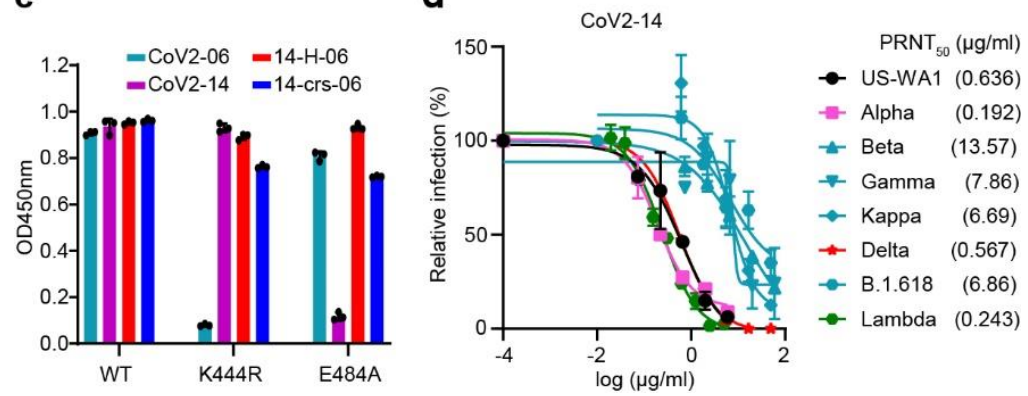

776 Extended Data Fig. 3. Additional binding and neutralizing characterizations of antibody

777 against the variants. (a-b) Neutralizations of SARS-CoV-2 virus with K444R mutation (a)

778 and E484A mutation (b) by CoV2-06 and CoV2-14. The assay is based on the mNeonGreen

779 reporter virus and the $\mathrm{NT}_{50}$ values are labeled. c, ELISA binding to the WT RBD and the K444R

780 and E484A RBD mutants by indicated antibodies. d, PRNT of CoV2-14 against the SARS-

781 CoV-2 US-WA1 strain and indicated SARS-CoV-2 variants. The PRNT50 values are labeled. 

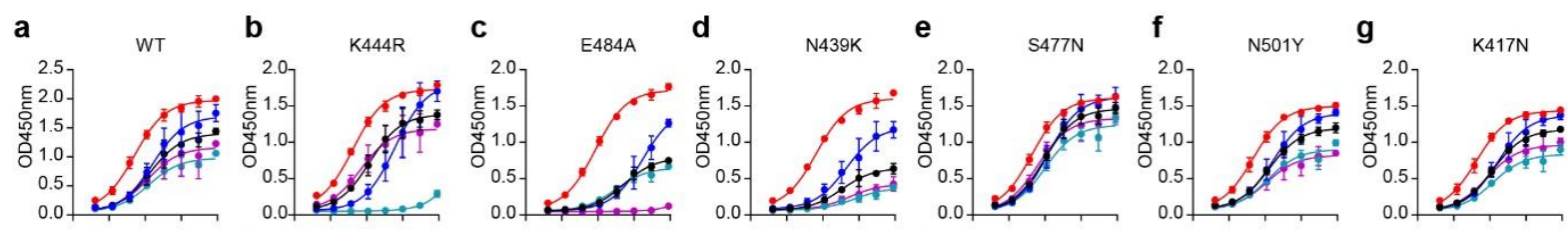

h

i $\mathrm{K} 417 \mathrm{~N}+\mathrm{E} 484 \mathrm{~K}+\mathrm{N} 501 \mathrm{Y}$
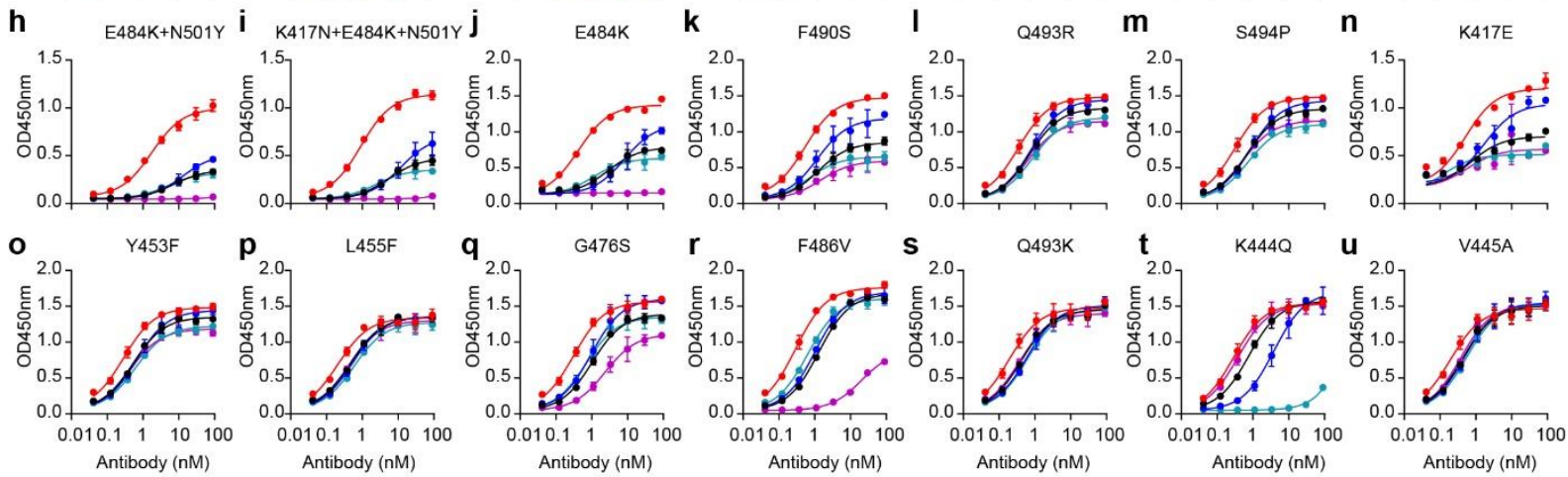

$\multimap$ 14-H-06 $\rightarrow$ 14crs06 cocktail $\rightarrow \lg \mathrm{C} 14 \rightarrow \lg \mathrm{G} 06$

Extended Data Fig. 4. ELISA bindings of bsAbs, individual antibodies and the cocktail to wild type and mutant RBDs. a-u, ELISA titrations of indicated antibodies to immobilized

WT RBD and RBD mutants. Data points are from duplicate wells. 
a

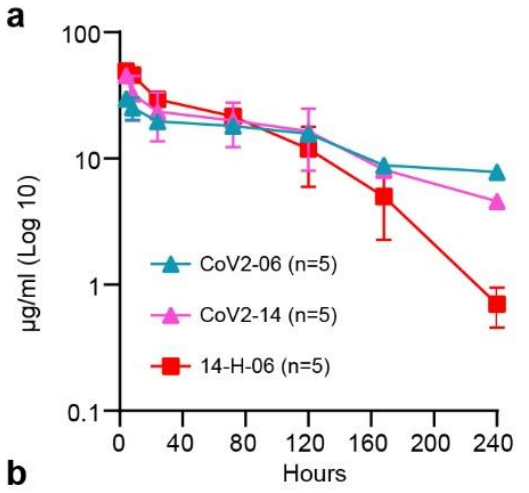

b

\begin{tabular}{|l|c|c|c|}
\hline & CoV2-06 & CoV2-14 & $14-\mathrm{H}-06$ \\
\hline T1/2 (hr.) & 137.40 & 74.72 & 29.02 \\
\hline AUC (hr/ug/ml) & 3438.63 & 3667.56 & 3533.27 \\
\hline
\end{tabular}

787 Extended Data Fig. 5. Antibody pharmacokinetics in mice. a, The serum concentrations of

788 injected antibodies at multiple time points (4, 8, 24, and 72 hours 5, 7, 10 days) post injection

789 were quantified by ELISA. b, Pharmacokinetics parameters were calculated by non-

790 compartmental analysis using Phoe $^{\mathrm{ni}} \mathrm{x} 64$ WinNonlin (8.3.3.33) software (Certara). 
791 Table S1. Data collection and refinement statistics for crystal structures

\begin{tabular}{|c|c|c|}
\hline & Fab06-RBD Complex & Fab14 \\
\hline Wavelength $(\AA)$ & 0.9464 & 0.9464 \\
\hline Resolution range $(\AA)^{\mathrm{a}}$ & $47.55-2.89(2.993-2.89)$ & $48.03-2.46(2.548-2.46)$ \\
\hline Space group & $\mathrm{P} 2{ }_{12} 2$ & $\mathrm{C} 2$ \\
\hline Unit cell a,b,c (̊) & $50.17,266.35,112.61$ & $77.7,71.22,96.76$ \\
\hline$\alpha, \beta, \gamma\left(^{\circ}\right)$ & $90,90,90$ & $90,99.07,90$ \\
\hline Total reflections & $363812(35178)$ & $130818(11319)$ \\
\hline Unique reflections & $34882(3389)$ & $18867(1696)$ \\
\hline Multiplicity & $10.4(10.4)$ & $6.9(6.7)$ \\
\hline Completeness (\%) & $99.81(98.86)$ & $98.83(89.35)$ \\
\hline Mean I/sigma(I) & $8.31(1.08)$ & $13.60(1.81)$ \\
\hline Wilson B-factor & 63.19 & 53.73 \\
\hline $\mathrm{R}_{\text {merge }}$ & $0.3153(2.504)$ & $0.1281(1.076)$ \\
\hline $\mathrm{R}_{\text {meas }}$ & $0.3316(2.632)$ & $0.1385(1.165)$ \\
\hline $\mathrm{R}_{\text {pim }}$ & $0.1006(0.7924)$ & $0.05213(0.4418)$ \\
\hline $\mathrm{CC}_{1 / 2}$ & $0.994(0.48)$ & $0.997(0.765)$ \\
\hline $\mathrm{CC}^{*}$ & $0.998(0.806)$ & $0.999(0.931)$ \\
\hline Reflections used in refinement & $34874(3388)$ & 18855 (1694) \\
\hline Reflections used for R-free & $1743(170)$ & $943(85)$ \\
\hline $\mathrm{R}_{\text {work }}^{\mathrm{b}}$ & $0.2287(0.3623)$ & $0.2104(0.3511)$ \\
\hline$R_{\text {free }}{ }^{b}$ & $0.2694(0.4140)$ & $0.2563(0.3683)$ \\
\hline $\mathrm{CC}_{\text {(work) }}$ & $0.938(0.656)$ & $0.948(0.795)$ \\
\hline $\mathrm{CC}_{(\text {free })}$ & $0.888(0.374)$ & $0.907(0.788)$ \\
\hline Number of non-hydrogen atoms & 9536 & 3384 \\
\hline protein & 9411 & 3310 \\
\hline NAG & 28 & \\
\hline water & 97 & 74 \\
\hline Protein residues & 1242 & 438 \\
\hline RMS(bonds) $(\AA)$ & 0.012 & 0.010 \\
\hline RMS(angles) $\left(^{\circ}\right)$ & 1.55 & 1.27 \\
\hline Ramachandran favored (\%) & 95.43 & 95.39 \\
\hline Ramachandran allowed (\%) & 4.57 & 4.61 \\
\hline Ramachandran outliers (\%) & 0.00 & 0.00 \\
\hline Rotamer outliers $(\%)$ & 0.66 & 0.00 \\
\hline Clashscore & 13.06 & 8.74 \\
\hline Average B-factor & 67.21 & 57.01 \\
\hline protein & 67.34 & 57.00 \\
\hline water & 49.33 & 57.17 \\
\hline
\end{tabular}


bioRxiv preprint doi: https://doi.org/10.1101/2022.02.01.478504; this version posted February 1,2022 . The copyright holder for this preprint

(which was not certified by peer review) is the author/funder, who has granted bioRxiv a license to display the preprint in perpetuity. It is made available under aCC-BY-NC-ND 4.0 International license.

793 Table S2. Engineered mutations in the spike region of recombinant SARS-CoV-2 variants.

\begin{tabular}{|c|c|}
\hline Variants & Spike mutations \\
\hline $\begin{array}{l}\text { Alpha } \\
(B .1 .1 .7)\end{array}$ & $\Delta 69-70, \Delta 145, \mathrm{~N} 501 \mathrm{Y}, \mathrm{A} 570 \mathrm{D}, \mathrm{D} 614 \mathrm{G}, \mathrm{P} 681 \mathrm{H}, \mathrm{T} 716 \mathrm{I}, \mathrm{S} 982 \mathrm{~A}$, and D1118H \\
\hline $\begin{array}{l}\text { Beta } \\
(\text { B.1.351) }\end{array}$ & D80A, D215G, $4242-244, \mathrm{~K} 417 \mathrm{~N}, \mathrm{E} 484 \mathrm{~K}, \mathrm{~N} 501 \mathrm{Y}, \mathrm{D} 614 \mathrm{G}$, and A701V \\
\hline $\begin{array}{l}\text { Gamma } \\
(\text { P.1) }\end{array}$ & $\begin{array}{l}\text { L18F, T20N, P26S, D138Y, R190S, K417T, E484K, N501Y,D614G, H655Y, T1027I, and } \\
\text { V1176F }\end{array}$ \\
\hline $\begin{array}{l}\text { Kappa } \\
(B .1 .617 .1)\end{array}$ & G142D, E154K, L452R, E484Q, D614G, P681R, Q1071H, and H1101D \\
\hline $\begin{array}{l}\text { Delta } \\
(B .1 .617 .2)\end{array}$ & T19R, G142D, L452R, T478K, D614G, P681R, and D950N \\
\hline Lambda & G75V, T76I, $\Delta 246-252$, D253N, L452Q, F490S, D614G, and T859N \\
\hline B.1.618 & $\mathrm{H} 49 \mathrm{Y}, \Delta 145-146, \mathrm{E} 484 \mathrm{~K}$, and D614G \\
\hline $\begin{array}{l}\text { Omicron } \\
\text { (B.1.1.529) }\end{array}$ & $\begin{array}{l}\text { A67V, del69-70, T95I, } \Delta 142-144, \text { Y145D, del211, L212I, ins214EPE, G339D, S371L, S373P, } \\
\text { S375F, K417N, N440K, G446S, S477N, T478K, E484A, Q493R, G496S, Q498R, N501Y, } \\
\text { Y505H, T547K, D614G, H655Y, N679K, P681H, N764K, D796Y, N856K, Q954H, N969K, } \\
\text { L981F }\end{array}$ \\
\hline
\end{tabular}

